\title{
Specific Detection and Identification of Fusarium graminearum Sensu Stricto Using a PCR-RFLP Tool and Specific Primers Targeting the Translational Elongation Factor $1 \alpha$ Gene
}

\author{
Mohamed Hafez, ${ }^{1,2}$ Ahmed Abdelmagid, ${ }^{1,3}$ Lorne R. Adam, ${ }^{1}$ and Fouad Daayf ${ }^{1, \dagger}$ \\ ${ }^{1}$ Department of Plant Science, University of Manitoba, Winnipeg, Manitoba, R3T2N2, Canada \\ ${ }^{2}$ Department of Botany and Microbiology, Faculty of Science, Suez University, Suez, Egypt \\ ${ }^{3}$ Department of Plant Pathology, Assiut University, Assiut,71515, Egypt
}

\begin{abstract}
Fusarium graminearum is a toxigenic plant pathogen that causes Fusarium head blight (FHB) disease on cereal crops. It has recently shown to have cross-pathogenicity on noncereals (i.e., Fusarium root rot [FRR] on soybean) in Canada and elsewhere. Specific detection and differentiation of this potent toxigenic, trichothecene-producing pathogen among other closely related species is extremely important for disease control and mycotoxin monitoring. Here, we designed a PCR restriction fragment length polymorphism protocol based on the DNA sequence of the translational elongation factor $1 \alpha(T E F 1 \alpha)$ gene. A unique restriction site to the enzyme $H p a I I$ is only found in $F$. graminearum sensu stricto strains among differ-

$\mathrm{mh} 1 / \mathrm{mh} 2$ generated a 459-bp PCR fragment. Restriction digestion of the generated fragments with the HpaII enzyme generated a unique restriction pattern that can rapidly and accurately differentiate $F$. graminearum sensu stricto among all other Fusarium spp. A primer pair (FgssF/FgssR) specific to $F$. graminearum sensu stricto also was designed and can distinguish $F$. graminearum sensu stricto from all other Fusarium spp. in the FGSC and other closely related Fusarium spp. involved in FHB and FRR. This finding will be very useful for the specific detection of $F$. graminearum sensu stricto for diagnostic purposes as well as for the accurate detection of this pathogen in breeding and other research purposes.
\end{abstract} ent Fusarium strains in the $F$. graminearum species complex (FGSC) and other Fusarium spp. associated with FHB in cereals and FRR in soybean. Partial amplification of the $T E F 1 \alpha$ gene with newly designed primers
Keywords: detection, Fusarium graminearum species complex, PCRRFLP, species-specific primers
Fusarium spp. are potent plant pathogens that cause several economically important diseases such as Fusarium head blight (FHB) and crown rot in cereals. Fusarium graminearum has been recently reported as a major necrotrophic pathogen of soybean causing seed decay, pre- and postemergence damping-off, pod blight, and Fusarium root rot (FRR) in Canada and other regions. Cross-pathogenicity of $F$. graminearum and other Fusarium spp. between cereal and soybean or pulse crops has been recently reported (Broders et al. 2007; Chiotta et al. 2016; Dill-Macky and Jones 2000; Sella et al. 2014; Xue et al. 2007). FHB in cereals is caused by several species of Fusarium, including $F$. graminearum, $F$. avenaceum, $F$. cerealis, $F$. poae, and $F$. sporotricioides; however, $F$. graminearum is the primary species involved. FRR in soybean and pulse crops is caused by several Fusarium spp., most commonly by $F$. oxysporum and $F$. solani (Gray and Achenbach 1996; Roy 1997). Other Fusarium spp. have been reported as FRR-causative agents in soybean and pulse crops, including $F$. redolens, F. acuminatum, F. chlamydosporum, F. culmorum, F. equiseti, $F$. incarnatum, and $F$. graminearum (Bienapfl et al. 2010; Kulik et al.

Current address of M. Hafez: Agriculture and Agri-Food Canada, Lethbridge Research and Development Centre, Lethbridge, Alberta, T1J 4B1, Canada.

${ }^{\dagger}$ Corresponding author: F. Daayf; daayff@cc.umanitoba.ca

Funding: This work was supported by the Manitoba Pulse and Soybean Growers Association; the Manitoba Wheat and Barley Growers Association; and the Canadian MITACS-Accelerate internship program, which partially covered the salary of M. Hafez.

*The $\boldsymbol{e}$-Xtra logo stands for "electronic extra" and indicates that two supplementary figures and two supplementary tables are published online.

The author(s) declare no conflict of interest.

Accepted for publication 18 November 2019.

() 2020 The American Phytopathological Society
2004; Lu et al. 2015; Zitnick-Anderson et al. 2018). Recently, there has been first report of the FHB pathogen $F$. cerealis causing root rot in soybean (Abdelmagid et al. 2018).

Some plant diseases can be managed by reducing the inoculum potential for future growing seasons. Inoculum can be reduced by eliminating the pathogen's spore-producing structures (Dill-Macky and Jones 2000) or removing the susceptible hosts species in a withinfield crop rotation (Guo et al. 2010; Marburger et al. 2015). Crop rotation can be an effective and inexpensive method for managing some plant diseases but it is not necessarily effective for foliar or aerial diseases such as FHB in cereals. Nevertheless, because Fusarium spp. causing FHB (i.e., F. cerealis) were found to cause root rots in other crops such as soybean (Abdelmagid et al. 2018), rotation may contribute, at least in part, to FHB mitigation. Control of the disease is achieved by growing nonhost crops until the pathogen in the soil dies or its population decreases to a low enough level that there is negligible crop damage. Successful disease control by crop rotation requires complete understanding of the pathogen's life cycle. Some pathogens such as Fusarium spp. can survive in the soil for a long time even in the absence of its original host. Fusarium spp. can survive in the soil as a saprophyte on plant debris, and can also persist for many years as chlamydospores (Dill-Macky and Jones 2000; Lecomte et al. 2016; Marburger et al. 2015). Rotating wheat with soybean is one of the most frequently used crop rotation regimes in Canada, the United States, and other countries. Wheat offers a number of significant benefits when grown with soybean. It helps in controlling important pests and depletes the number of soybean cyst nematodes in the rhizosphere (Baird and Bernard 1984; Bajaj et al. 2015; Liebman and Dyck 1993; Schreiber 1992; Sindelar et al. 2016). F. graminearum is the most dominant Fusarium sp. that causes FHB in wheat and other cereal crops such as barley and oat around the world (McMullen et al. 1997). This pathogen can cause yield loss and grain contamination with trichothecene mycotoxins such as deoxynivalenol (DON) and its acetylated forms, 3ADON or 15ADON (Goswami and Kistler 2004; McMullen et al. 1997). $F$. graminearum was also shown to be a soybean pathogen causing seed decay, damping-off, and root rot (Broders et al. 2007; Ellis 
et al. 2011; Martinelli et al. 2004; Pioli et al. 2004). The transport of plant pathogens through the atmosphere involves three stagesliberation, drift, and deposition-and these stages are influenced by ecological and environmental factors (Schmale and Ross 2015). Abiotic factors (temperature, relative humidity, light, and wind) also play an important role in the spread of plant pathogens and the impact of these factors on the release of $F$. graminearum spores was previously investigated (David et al. 2014; Trail et al. 2002).

The $F$. graminearum species complex (FGSC) contains at least 16 phylogenetically distinct species (O'Donnell et al. 2000, 2008; Starkey et al. 2007; Yli-Mattila et al. 2009). It is extremely difficult to discriminate between members within the FGSC using morphological or microscopy-based methods. However, several molecular markers have been developed based on genetic differences for discerning species identity (Wang et al. 2011). Culture-based methods for identifying plant pathogens are time consuming and require extensive knowledge of classical taxonomy. Molecular methods have replaced the traditional phenotypic methods for accurate species identification, especially with morphologically and microscopically cryptic species such as Fusarium spp. Several PCR-based methods were developed for detection of species in the FGSC (Biazio et al. 2008; Jurado et al. 2005; Nicholson et al. 1998; Niessen 2007; Niessen and Vogel 1997; Schilling et al. 1996; Tan and Niessen 2003). These methods were designed to detect members in the FGSC ( $F$. graminearum sensu lato) but none can specifically detect $F$. graminearum sensu stricto. Members of the FGSC are considered to be the main causative agent of FHB of wheat, barley, oat, and other small cereal grain crops worldwide. Many mycotoxins could be produced by FGSC, with trichothecenes being the most prevalent Fusarium mycotoxins in cereal grains; they are harmful to human and animal health, thereby posing a serious threat to food and feed safety (Aoki et al. 2012; Sudakin 2003). Trichothecenes are divided into four major groups based on their chemical structure: type A, type B, type C, and type D. Among these, type B trichothecenes are the most commonly detected mycotoxin in cereal grains and their related products (Placinta et al. 1999). Proper species identification is critical to any research aimed at improving disease and mycotoxins control. Accurate species identification for members of FGSC is highly important due to the ability of FGSC to produce many trichothecene mycotoxins, especially those from type B such as nivalenol (NIV) and DON, in addition to their acetylated derivatives 4ANIV, 3ADON, and 15ADON (van der Lee et al. 2015).

The $\mathrm{Fg} 16 \mathrm{~F} / \mathrm{R}$ primer pair was originally designed to detect trichothecene-producing and nonproducing isolates of $F$. graminearum colonizing wheat grains (Nicholson et al. 1998). It targets the Fgl6 gene that is located in chromosome 1 in F. graminearum and other species in the FGSC, the gene codes for a hypothetical protein whose function is not yet known. Recently, it was found that there is a possible link between the $\mathrm{Fg} 16$ gene and mitochondrial lipid oxidation (Guenther et al. 2009). Guenther and Trail (2005) studied gene expression during perithecium development both in planta and in vitro and they found that lipid oxidation occurs as perithecia are developing. Sequence-characterized amplified region (SCAR) analysis using the $\mathrm{Fg} 16 \mathrm{~F} / \mathrm{R}$ primer pair was previously used to detect and differentiate between different species within the FGSC (Carter et al. 2002). However, differentiation of some species in the FGSC on the basis of SCAR fragment size was not reliable because this primer pair produces a polymorphic band for FGSC isolates $F$. cortaderiae, $F$. brasilicum, $F$. meridionale, $F$. asiaticum, $F$. ussurianum, $F$. louisianense, and F. mesoamericanum (approximately $500 \mathrm{bp}$ ). F. graminearum, $F$. austroamericanum, $F$. boothii, $F$. acaciae-mearnsii, $F$. nepalense, $F$. gerlachii, $F$. aethiopicum, and $F$. vorosii produce amplicons smaller than 500 bp (Garmendia et al. 2018). F. graminearum, $F$. nepalense, $F$. gerlachii, and $F$. aethiopicum produce identical amplicons when amplified with $\mathrm{Fg} 16 \mathrm{~F}$ and $\mathrm{Fg} 16 \mathrm{R}$ primers. Another FGSC-specific SCAR primer pair is UBC85F/R (Schilling et al. 1996), which produces monomorphic amplicons (332 bp) with all species in the FGSC. Neither the Fg16F/R nor UBC85F/R primer pairs are able to differentiate $F$. graminearum sensu stricto from other Fusarium spp. in the FGSC. Recently, a PCR restriction fragment length polymorphism (RFLP) method was reported based on the translational elongation factor $1 \alpha(T E F 1 \alpha)$ gene to differentiate $F$. graminearum sensu stricto from other species within the FGSC (Garmendia et al. 2018). This PCR-RFLP tool was designed to amplify the $T E F 1 \alpha$ gene with the commonly used ef $1 / 2$ primer pair and digest the amplified PCR fragments with $B s a \mathrm{HI}$ enzyme. The multiple recognition sites for BsaHI (5'-GRCGYC-3'; GACGCC, GACGTC, GGCGCC, and GGCGTC) affect its specificity to $F$. graminearum sensu stricto and other Fusarium spp. (F. asiaticum, $F$. pseudograminearum, and $F$. chlamydosporum) and can be recognized and cleaved by the BsaHI enzyme.

Several genes have been used to define species boundaries within the genus Fusarium: internal transcribed spacer (ITS) region (Edel et al. 1997; Schoch et al. 2012; Zarrin et al. 2016), mating type locus (Kashyap et al. 2015; Starkey et al. 2007), phosphate permease (Ortiz et al. 2017; Stakheev et al. 2016), trichothecene-3-O-acetyltransferase (Garvey et al. 2008; Kimura et al. 1998), and $\beta$-tubulin ( $\beta-T U B$ ) (Mach et al. 2004). However, the TEF1 $\alpha$ gene has been the most frequently used sequence to define species boundaries within the genus Fusarium (Carbone and Kohn 1999; Garmendia et al. 2018; Geiser et al. 2004; Knutsen et al. 2004; Kristensen et al.2005; Mbofung et al. 2007; O’Donnell et al. 1998, 2008; Zitnick-Anderson et al. 2018).

The objective of this work was to design molecular markers for specific detection of $F$. graminearum sensu stricto for rapid and accurate detection of this pathogen among other Fusarium spp. in the FGSC and other Fusarium spp. associated with FHB and FRR diseases in cereal crops and soybean, respectively.

\section{Materials and Methods}

Fungal cultures. Fusarium spp. isolated from several plant hosts (soybean, pea, wheat, barley, and oat) in Manitoba, Canada, were used in the current study. Wheat, barley, and oat Fusarium isolates were provided by Dr. Xiben Wang and Dr. Maria-Antonia Henriquez from Morden Research Center, Agriculture and Agri-Food Canada (AAFC, Morden, MB, Canada). Some soybean and pea Fusarium isolates were provided by Dr. Debra McLaren from Brandon Research Centre (AAFC, Brandon, MB, Canada). The remaining soybean Fusarium isolates as well as other plant pathogens such as Colletotrichum spaethianum, Rhizoctonia solani, Sclerotinia sclerotiorum, and Diaporthe caulivora were collected and characterized by colleagues in our lab at the Plant Science Department, University of Manitoba, Canada. Nine species from the FGSC were obtained from the culture collection of United States Department of Agriculture-Agricultural Research Service (USDA-ARS, Peoria, IL, U.S.A.). The Fusarium isolates were initially identified based on morphological and microscopic characteristics and confirmed based on the TEF $1 \alpha$ gene sequence (see Table 1 for the complete species list).

DNA extraction and PCR amplification. Genomic DNA (gDNA) was extracted from single-spore cultures of each fungus using the GenElute plant gDNA miniprep kit (Sigma-Aldrich) based on the manufacturer's recommendations. The primer pairs ef1/ef2 and $\mathrm{mh} 1 / \mathrm{mh} 2$ were used to amplify two fragments from the TEF $1 \alpha$ gene; primers and binding positions of these primers are shown in Figure 1 and Table 2. PCRs were performed in a final volume of $50 \mu \mathrm{l}$ using the DreamTaq Green PCR Master Mix (Thermo Fisher Scientific, Canada) with the following reagent concentrations: Green Taq buffer $(1 \times)$, dNTP mixture (200 $\mu \mathrm{M}$ each), forward and reverse primers $(0.2 \mu \mathrm{M}$ each), Dream Taq DNA polymerase (1.5 U per $50 \mu \mathrm{l})$, approximately 20:30 ng of gDNA template, and the total volume of the PCR adjusted to $50 \mu$ l with nuclease-free $\mathrm{H}_{2} \mathrm{O}$. PCR amplification included an initial denaturation step at $94^{\circ} \mathrm{C}$ for $3 \mathrm{~min}$; followed by 35 cycles of $94^{\circ} \mathrm{C}$ for $1 \mathrm{~min}, 50^{\circ} \mathrm{C}$ for $50 \mathrm{~s}$, and $72^{\circ} \mathrm{C}$ for $1 \mathrm{~min}$; and a final extension at $72^{\circ} \mathrm{C}$ for $5 \mathrm{~min}$.

The Fg16 primer pair ( Fg16F/R) was used for specific detection of the FGSC isolates. The PCR master mix was as previously mentioned above for the TEF1 $\alpha$ gene. The PCR amplification was performed according to Nicholson et al. (1998). FGSC-specific PCR detection with the UBC85F/R primer pair was performed according 
to Schilling et al. (1996), with a PCR master mix as described for the TEF $1 \alpha$ gene.

For specific detection of $F$. graminearum sensu stricto, two species-specific primers (FgssF and FgssR) were designed to have a $3^{\prime}$ end unique to $F$. graminearum sensu stricto isolates exactly at the HpaII restriction site (Fig. 1). The FgssF primer was paired with ef2, while the FgssR primer was paired with FusF. The FusF primer is modified from the universal ef 1 primer designed by O'Donnell et al. (1998) but with higher melting temperature (Tm) for optimum pairing with FgssR primer. These two primer pairs were used to detect $F$. graminearum sensu stricto and differentiate this pathogen among other Fusarium spp. as well as other pathogens associated with wheat and soybean. PCRs were performed in a final volume of $50 \mu \mathrm{l}$ using the DreamTaq Green PCR Master Mix (Thermo Fisher Scientific, Canada) with the following reagent concentrations: Green Taq buffer $(1 \times)$, dNTP mixture $(200 \mu \mathrm{M}$ each), forward and reverse primers $(0.2 \mu \mathrm{M}$ each), Dream Taq DNA polymerase (1.5 U per $50 \mu \mathrm{l}$ ), approximately $10 \mathrm{ng}$ of gDNA template, and the total volume of the PCR adjusted to $50 \mu \mathrm{l}$ with nuclease-free $\mathrm{H}_{2} \mathrm{O}$. Touchdown PCR protocol was utilized to insure primer specificity using an initial annealing temperature (Ta) higher than the optimal Tm of the primers and gradually reducing over subsequent cycles until the optimal Ta temperature was reached. PCR amplification included an initial denaturation step of $95^{\circ} \mathrm{C}$ for $3 \mathrm{~min}$; followed by $1 \mathrm{~min}$ at $95^{\circ} \mathrm{C}, 30 \mathrm{~s}$ at Ta, and $1 \mathrm{~min}$ at $72^{\circ} \mathrm{C}$; and a final extension for $5 \mathrm{~min}$ at $72^{\circ} \mathrm{C}$. The initial Ta used with the FgssF/ef2 primer pair was $53^{\circ} \mathrm{C}$ for the first 5 cycles and $52^{\circ} \mathrm{C}$ for the next 5 cycles followed by $51^{\circ} \mathrm{C}$ for the last $25 \mathrm{cy}-$ cles. The initial Ta used with the FusF/FgssR primer pair was $57^{\circ} \mathrm{C}$ for the first 5 cycles and $56^{\circ} \mathrm{C}$ for the next 5 cycles followed by $55^{\circ} \mathrm{C}$ for the last 25 cycles.

Specific detection of $F$. graminearum sensu stricto with FgssF/ef2 and FusF/FgssR primer pairs was also performed with Phusion highfidelity (HF) DNA polymerase (Thermo Fisher Scientific, Canada). PCRs were performed in a final volume of $20 \mu \mathrm{l}$ at the following reagent concentrations: $5 \times$ Phusion HF Green buffer $(4 \mu \mathrm{l}), 10 \mathrm{mM}$ dNTP mixture $(0.4 \mu \mathrm{l}), 10 \mu \mathrm{M}$ forward and reverse primers $(1 \mu \mathrm{l}$ each), Phusion HF DNA polymerase $(0.2 \mu \mathrm{l})$ at $2 \mathrm{U} / \mu \mathrm{l}$, approximately
10 ng of gDNA template, and the total volume of the PCR adjusted to $20 \mu \mathrm{l}$ with nuclease-free $\mathrm{H}_{2} \mathrm{O}$. PCR amplification included an initial denaturation step at $98^{\circ} \mathrm{C}$ for $30 \mathrm{~s}$; followed by 30 cycles of $98^{\circ} \mathrm{C}$ for $10 \mathrm{~s}$, Ta for $15 \mathrm{~s}$, and $72^{\circ} \mathrm{C}$ for $20 \mathrm{~s}$; and a final extension at $72^{\circ} \mathrm{C}$ for $5 \mathrm{~min}$. Ta for FgssF/ef2 was $59^{\circ} \mathrm{C}$ and for FusF/FgssR was $63^{\circ} \mathrm{C}$

Trichothecene genotyping based on Tri12 multiplex PCR. Genotype determination of type-B trichothecene-producing Fusarium spp. was performed according to Ward et al. (2008) based on sequence diversity on the Tri12 gene (encodes a trichothecene efflux pump). Tri12 multiplex PCR produced amplicons of approximately 840, 670, and 410 bp with NIV, 15ADON, and 3ADON genotypes, respectively. PCRs for Tri12 multiplex PCR were performed in a final volume of $25 \mu \mathrm{l}$ using DreamTaq (Thermo Fisher Scientific, U.S.A.), with the following reagent concentrations: Taq green buffer $(1 \times)$; dNTP mixture (200 $\mu \mathrm{M}$ each); 12CON, 12NF, 12-15F, and 12$3 \mathrm{~F}$ primers $(0.2 \mu \mathrm{M}$ each); DreamTaq DNA polymerase (1.5 U per

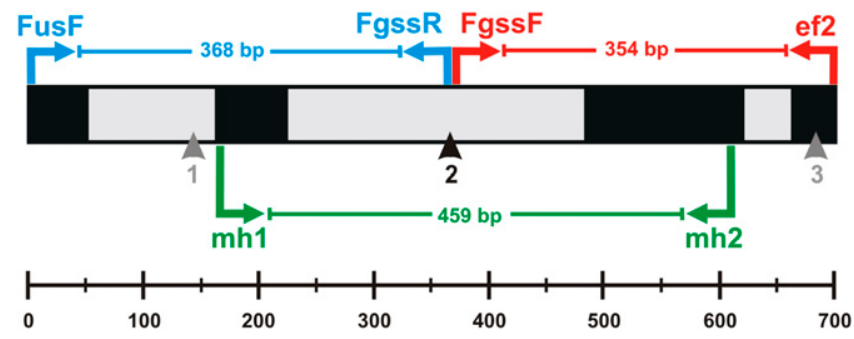

Fig. 1. Schematic representation of the translational elongation factor $1 \alpha$ gene showing the exons (black boxes) and introns (gray boxes) and the binding sites of primers. The ef1/ef2 PCR fragment contains three Hpall restriction sites (5'-CCGG$3^{\prime}$ ); restriction sites 1 and 3 (gray triangles) are found in all investigated Fusarium spp., whereas restriction site 2 (black triangle) is found only in Fusarium graminearum sensu stricto and absent in all other Fusarium spp., including the $F$. graminearum species complex. The Hpall restriction site is found in intron number 2: 199 nucleotides from the mh1 primer and 260 nucleotides from the mh2 primer.

Table 1. List of Fusarium spp. used during the current study, along with translational elongation factor $1 \alpha$ (TEF1 $\alpha$ ) GenBank accession numbers, plant host, and country of origin ${ }^{\mathrm{a}}$

\begin{tabular}{|c|c|c|c|c|c|c|}
\hline Species & Isolate & $T E F 1 \alpha$ accession & Host & Country & FGSC & Type-B \\
\hline Fusarium oxysporum & Carm31 & MH315926 & Soybean & Canada & No & No \\
\hline F. redolens & MLT5 & МH315933 & Soybean & Canada & No & No \\
\hline F. avenaceum & HSW15.93 & MH315905 & Wheat & Canada & No & No \\
\hline F. acuminatum & Carm2 & MH315914 & Soybean & Canada & No & No \\
\hline F. equiseti & Carm35 & MH315930 & Soybean & Canada & No & No \\
\hline F. incarnatum & Carm37 & MH479893 & Soybean & Canada & No & No \\
\hline F. poae & MRC99 & MH315911 & Oat & Canada & No & No \\
\hline F. culmorum & MLT10 & МH315937 & Soybean & Canada & No & 3ADON \\
\hline F. cerealis & Carm17 & MH151080 & Soybean & Canada & No & NIV \\
\hline F. cerealis & Carm20 & MH151081 & Soybean & Canada & No & NIV \\
\hline F. graminearum & HSW15.16 & MH315907 & Wheat & Canada & Yes & $3 \mathrm{ADON}$ \\
\hline F. graminearum & MRC85 & MH315909 & Oat & Canada & Yes & 3ADON \\
\hline F. graminearum & MRC31 & MH315910 & Oat & Canada & Yes & $15 \mathrm{ADON}$ \\
\hline F. graminearum & Carm3 & MH315915 & Soybean & Canada & Yes & $15 \mathrm{ADON}$ \\
\hline F. graminearum & Carm10 & MH315922 & Soybean & Canada & Yes & $15 \mathrm{ADON}$ \\
\hline F. mesoamericanum & NRRL 25797 & AF212441 & Banana & Honduras & Yes & NIV \\
\hline F. acaciae-mearnsii & NRRL 26752 & AF212447 & Acacia mearnsii & South Africa & Yes & NIV \\
\hline F. aethiopicum & NRRL 46710 & FJ240295 & Wheat & Ethiopia & Yes & $15 \mathrm{ADON}$ \\
\hline F. boothii & NRRL 26916 & GQ915503 & Corn & South Africa & Yes & $15 \mathrm{ADON}$ \\
\hline F. brasilicum & NRRL 31281 & AY452964 & Oat & Brazil & Yes & 3ADON \\
\hline F. gerlachii & NRRL 36905 & DQ459742 & Orchard grass & New Zealand & Yes & NIV \\
\hline F. meridionale & NRRL 28436 & AF212435 & Orange twig & New Caledonia & Yes & NIV \\
\hline F. nepalense & NRRL 54220 & KM889629 & Rice & Nepal & Yes & $15 \mathrm{ADON}$ \\
\hline F. vorosii & NRRL 37605 & DQ459745 & Wheat & Hungary & Yes & $15 \mathrm{ADON}$ \\
\hline
\end{tabular}

${ }^{a}$ Fusarium spp. belonging to F. graminearum species complex (FGSC) are indicated. Type-B trichothecene (Type-B) genotype based on Tri12 multiplex PCR (nivalenol [NIV], 3-acetyl-deoxynivalenol [3ADON], or 15-acetyl-deoxynivalenol [15ADON]) for Fusarium spp. used in the current study are also indicated. F. pseudograminearum NRRL40886 was also tested during the current study. 
$50 \mu \mathrm{l}$ ); approximately 20:30 ng of gDNA template; and the total volume of the PCR adjusted to $25 \mu \mathrm{l}$ with nuclease-free $\mathrm{H}_{2} \mathrm{O}$. Detailed information about the primers used in Tri12 multiplex PCR is shown in Table 2. PCR amplification conditions for Tri12 multiplex PCR included an initial denaturation step at $94^{\circ} \mathrm{C}$ for $2 \mathrm{~min}$; followed by 30 cycles of $94^{\circ} \mathrm{C}$ for $30 \mathrm{~s}, 52^{\circ} \mathrm{C}$ for $30 \mathrm{~s}$, and $72^{\circ} \mathrm{C}$ for $1 \mathrm{~min}$; and a final extension at $72^{\circ} \mathrm{C}$ for $5 \mathrm{~min}$.

All PCR amplicons were analyzed by gel electrophoresis through 1 to $2 \%$ agarose gels in $1 \times$ Tris-borate-EDTA (TBE) buffer $(89 \mathrm{mM}$ Tris-borate and $10 \mathrm{mM}$ EDTA, $\mathrm{pH}$ 8.0). The sizes of the PCR amplicons were estimated against a 1-kb-plus DNA ladder (Thermo Fisher Scientific, Canada) and visualized under UV light after staining with ethidium bromide $(5 \mu \mathrm{g} / \mathrm{ml})$. PCR products were purified with the Wizard SV Gel and PCR Clean-Up System (Promega, Canada) and sent for sequencing (Macrogen, Rockville, MD, U.S.A.).

Sequence analysis. Individual TEF1 $\alpha$ gene sequences were compiled and assembled manually into contigs using the GeneDoc program, v2.5.010 (Nicholas et al. 1997). The initial nucleotide sequence alignments were done with the Clustal-X program (Thompson et al. 1997) and the resulting alignments were refined by eye with the GeneDoc program, v2.5.010. The online resource Basic Local Alignment Search Tool (https://www.ncbi.nlm.nih.gov/BLAST/) (Altschul et al. 1990) was used to retrieve similar sequences from the NCBI database to have significant representatives from each species investigated in the current study as well as sequences that represent other Fusarium spp. involved in FHB and FRR diseases. A phylogenetic tree was constructed using the iTOL v3 online tool (Letunic and Bork 2016) based on TEF $1 \alpha$ sequences (see Supplementary Table

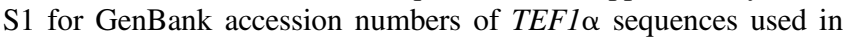
the tree).

PCR-RFLP based on TEF1 $\alpha$ gene sequence. TEF $1 \alpha$ sequences for each Fusarium spp. were analyzed separately with the RestrictionMapper program (version 3; http://www.restrictionmapper.org/). The HpaII restriction enzyme was chosen because it provided species-specific restriction pattern for $F$. graminearum sensu stricto. Based on sequence alignment for the $T E F 1 \alpha$ gene, the restriction site for HpaII (5'-CCGG-3') was found in all tested Fusarium spp. at two positions and found in $F$. graminearum sensu stricto at three positions (Fig. 1). A new primer pair, $\mathrm{mh} 1 / \mathrm{mh} 2$, that binds conserved sequences in all Fusarium spp. was designed to amplify a fragment from the TEF1 $\alpha$ gene in all Fusarium spp. This mh1/mh2 PCR fragment containing the HpaII restriction site is only found in $F$. graminearum sensu stricto and not in the FGSC strains or other Fusarium strains tested. Restriction digestion of the $\mathrm{mh} 1 / \mathrm{mh} 2$ fragment was selected to give a unique pattern for all $F$. graminearum sensu stricto isolates, while any other Fusarium spp. DNA in that region will not be cut by the HpaII enzyme and gives a different restriction pattern.
Restriction digestions for mh1/mh2-TEF1 $\alpha$ PCR amplicons with HpaII enzyme were performed with approximately $400 \mathrm{ng}$ of the $\mathrm{mh} 1 / \mathrm{mh} 2 \mathrm{PCR}$ products $(10 \mu \mathrm{l})$ in a total volume of $30 \mu \mathrm{l}$, with $10 \times$ Tango buffer $(2 \mu \mathrm{l})$ and $10 \mathrm{U}$ of HpaII (Thermo Fisher Scientific, Canada) at $37^{\circ} \mathrm{C}$. Time needed for complete digestion of the $\mathrm{mh} 1 / \mathrm{mh} 2$ PCR amplicons by HpaII was tested by incubating the restriction mixture (described above) for 5, 10, 20, 30, and $60 \mathrm{~min}$. Restriction digestions for $\mathrm{mh} 1 / \mathrm{mh} 2-\mathrm{TEF} 1 \alpha$ PCR amplicons was also performed using FastDigest HpaII enzyme (Thermo Fisher Scientific, Canada) with approximately $400 \mathrm{ng}$ of the $\mathrm{mh} 1 / \mathrm{mh} 2 \mathrm{PCR}$ products $(10 \mu \mathrm{l})$ in $10 \times$ FastDigest Green buffer $(2 \mu \mathrm{l})$, and $2 \mu \mathrm{l}$ of FastDigest HpaII (Thermo Fisher Scientific, Canada), with a total volume adjusted to $30 \mu \mathrm{l}$ with nuclease-free water for $5 \mathrm{~min}$ at $37^{\circ} \mathrm{C}$. The resulting fragments were separated by gel electrophoresis (2\% agarose) for $90 \mathrm{~min}$ at $95 \mathrm{~V}$ in $1 \times \mathrm{TBE}$ (pH8.2). The gels were stained with ethidium bromide and visualized on the UV transilluminator. The size of digested bands was determined by comparing them with a 1-kb-plus DNA ladder (Thermo Fisher Scientific, Canada).

In planta experiments. An important objective during this study was to test the newly developed PCR-RFLP marker to specifically detect $F$. graminearum sensu stricto in infected wheat and soybean plant tissues. $F$. graminearum sensu stricto (isolate Carm3) and $F$. cerealis (isolate Carm1) were used to inoculate wheat and soybean to produce FHB and FRR, respectively. Wheat seed (Muchmore) were planted in $15-\mathrm{cm}$ pots filled with sterilized ready-mix soil. They were grown in a greenhouse at approximately $20 \pm 3^{\circ} \mathrm{C}$ with $16 \mathrm{~h}$ of supplemental light until flowering and anthesis formation. A signal central floret of the spikelet of the main stem was inoculated at anthesis with $10 \mu \mathrm{l}$ of macroconidial spore suspension $\left(5 \times 10^{4}\right.$ spores $/ \mathrm{ml}$ ) of $F$. graminearum sensu stricto Carm3 or $F$. cerealis Carm1 inoculated individually. Control plants were inoculated with $10 \mu l$ of sterile water. The spikes were covered with humid plastic bags for conidia germination and removed $36 \mathrm{~h}$ postinoculation. Plants were watered on odd days and treated with 20:20:20 nitrogen-phosphorus-potassium fertilizer and grown to full maturity. FHB symptoms were scored (nine spikes for each treatment) on a 0 -to-100\% scale, as described by Engle et al. (2003).

Soybean seed (22-60RY) were planted in ready-mix soil spiked with cornmeal $(20 \mathrm{~g} / \mathrm{kg}$ of soil) previously inoculated with a macroconidial suspension $\left(5 \times 10^{4}\right.$ spores $\left./ \mathrm{ml}\right)$ of $F$. graminearum sensu stricto Carm 3 or $F$. cerealis Carm 1 inoculated individually. Pots spiked with uninoculated cornmeal $(20 \mathrm{~g} / \mathrm{kg}$ of soil) were used as the control. Soybean plants were grown in a greenhouse at approximately $20 \pm 3^{\circ} \mathrm{C}$ with $16 \mathrm{~h}$ of supplemental light until the V3 growth stage and evaluated for root rot symptoms (nine plants for each treatment) on a 0-to-4 scale, as described by Esmaeili Taheri et al. (2011).

Surface-sterilized wheat grains and soybean roots from each treatment (three plants in each treatment) were plot dried, plated on potato

Table 2. List of primers used during the current study ${ }^{\mathrm{a}}$

\begin{tabular}{|c|c|c|c|c|c|}
\hline Primer & Sequence $\left(5^{\prime}\right.$ to $\left.3^{\prime}\right)$ & Direction & $\operatorname{Tm}\left({ }^{\circ} \mathbf{C}\right)$ & Target & Reference \\
\hline ef1 & ATGGGTAAGGARGACAAGAC & Forward & 52.7 & $T E F 1 \alpha$ & O'Donnell et al. 1998 \\
\hline ef2 & GGARGTACCAGTSATCATGTT & Reverse & 53.1 & $T E F 1 \alpha$ & O'Donnell et al. 1998 \\
\hline $\mathrm{Fg} 16 \mathrm{~F}$ & CTCCGGATATGTTGCGTCAA & Forward & 55 & $\mathrm{Fg} 16$ & Nicholson et al. 1998 \\
\hline $\mathrm{Fg} 16 \mathrm{R}$ & GGTAGGTATCCGACATGGCAA & Reverse & 56.6 & $\mathrm{Fg} 16$ & Nicholson et al. 1998 \\
\hline UBC85-F & GCAGGGTTTGAATCCGAGAC & Forward & 56.1 & SCAR85 & Schilling et al. 1996 \\
\hline UBC85-R & AGAATGGAGCTACCAACGGC & Reverse & 57.4 & SCAR85 & Schilling et al. 1996 \\
\hline $12 \mathrm{CON}$ & CATGAGCATGGTGATGTC & Reverse & 50.8 & Tril2 & Ward et al. 2008 \\
\hline $12-3 \mathrm{~F}$ & CTTTGGCAAGCCCGTGCA & Forward & 59.5 & Tri12 & Ward et al. 2008 \\
\hline $12-15 \mathrm{~F}$ & TACAGCGGTCGCAACTTC & Forward & 55.3 & Tri12 & Ward et al. 2008 \\
\hline $12 \mathrm{NF}$ & TCTCCTCGTTGTATCTGG & Forward & 50.5 & Tri12 & Ward et al. 2008 \\
\hline FusF & TGGGTAARGAGGASAAGACTCACC & Forward & 58.8 & $T E F 1 \alpha$ & This study \\
\hline $\operatorname{mh} 1$ & GGTCACTTGATCTACCAGTG & Forward & 52.4 & $T E F 1 \alpha$ & This study \\
\hline $\operatorname{mh} 2$ & GACAACATACCAATGACGGT & Reverse & 52.7 & $T E F 1 \alpha$ & This study \\
\hline FgssF & CTTTGTCGTAATTTTTTYCCC & Forward & 49.1 & $T E F 1 \alpha$ & This study \\
\hline FgssR & TATGAGCCCCACCGGG & Reverse & 57.1 & $T E F 1 \alpha$ & This study \\
\hline
\end{tabular}

${ }^{a}$ Melting temperature $(\mathrm{Tm})$, target gene or region (Target), and reference for each primer is indicated. $T E F 1 \alpha=$ translational elongation factor $1 \alpha$ and SCAR $=$ sequence-characterized amplified region. 
dextrose agar (PDA) medium, and incubated at 22 to $24^{\circ} \mathrm{C}$ for 7 days. Emerging Fusarium colonies from each treatment were purified and DNA was extracted from three Fusarium colonies in each treatment using the GenElute plant gDNA miniprep kit (Sigma-Aldrich, Canada) based on the manufacturer's recommendations. The TEF1 $\alpha$ gene was amplified by PCR (as described above) and sequenced for species identification. The extracted DNA (three replicates in each treatment) was used as a template in the PCR-RFLP protocol as described above to detect $F$. graminearum sensu stricto and $F$. cerealis within the infected wheat and soybean tissues and to differentiate $F$. graminearum sensu stricto from other species, especially the closely related $F$. cerealis pathogen.

\section{Results}

Fg16F/Fg16R and UBC85F/UBC85R amplification. The Fg16F/R primer pair is used to detect different Fusarium spp. within the FGSC. This primer pair produces PCR fragments with variable length depending on the species: $F$. brasilicum and $F$. meridionale produce a 500-bp fragment, while $F$. nepalense, $F$. gerlachii, and $F$. aethiopicum produce 400-bp fragments and $F$. vorosii produces a 390-bp fragment. All $F$. graminearum sensu stricto isolates investigated during the current study produce a 400-bp fragment when amplified with Fg16F and Fg16R primers. Some species within the FGSC did not produce any PCR amplicons (Fig. 2A, lanes 11, 17, and 24), and all other species outside the FGSC (Fig. 2A, lanes 1 to 10) gave negative results and did not produce any amplicons. Similarly, SCAR85 was amplified selectively from Fusarium spp. within the FGSC using UBC85F and UBC85R primers. The UBC85F/R primer pair produce a 332-bp PCR amplicon in some species of the FGSC (Fig. 2B, lanes 11 to 13, 17, 19 to 22, and 24), whereas some others within the FGSC (Fig. 2B, lanes 14 to 16, 18, and23) and other species not belonging to FGSC (Fig. 2B, lanes 1 to 10) showed no bands. Both Fg16F/R and UBC85F/R primer pairs can detect and differentiate species belonging to FGSC from any other Fusarium sp. but these primer pairs are not suitable to accurately differentiate between all species within the FGSC, and many false negatives can also be obtained. These two primer pairs were also not suitable for specific detection of $F$. graminearum sensu stricto.

PCR-RFLP for specific detection of $F$. graminearum sensu stricto. The HpaII restriction enzyme was chosen to develop a PCR-RFLP marker specific for $F$. graminearum sensu stricto because it provided a species-specific restriction pattern that can differentiate $F$. graminearum sensu stricto among all other Fusarium spp. Based on the alignment for the TEFI $\alpha$ sequences (approximately $700 \mathrm{bp}$; amplified with the ef1/ef2 primer pair), the restriction site for HpaII (5'-CCGG-3') was found in all investigated Fusarium spp. at two positions and found in $F$. graminearum sensu stricto at three positions (Fig. 1). Restriction digestion of the ef1/ef2 fragments
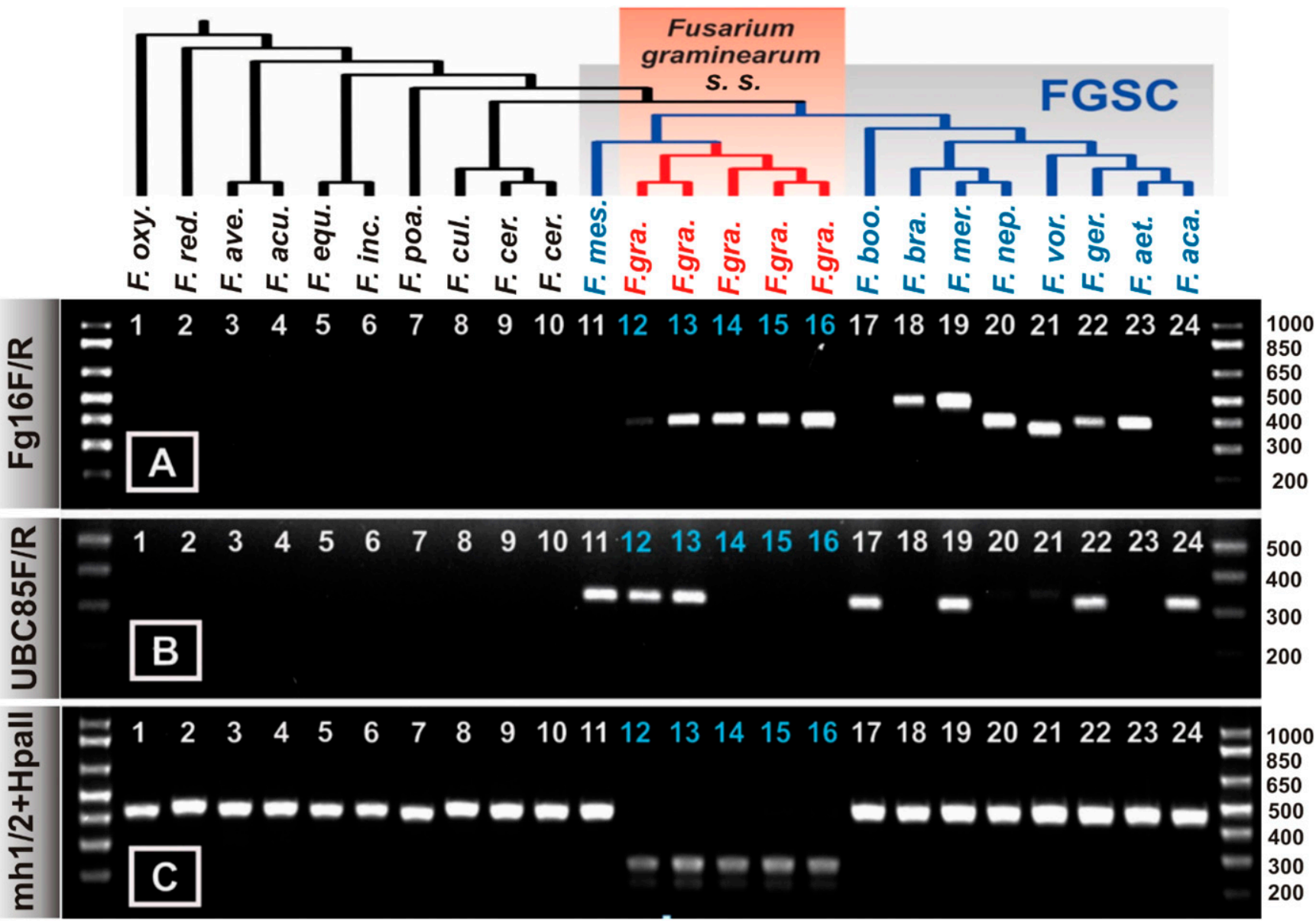

Fig. 2. Specific molecular detection of Fusarium graminearum sensu stricto. A, The commonly used Fg16F/R primer pair detects species within the $F$. graminearum species complex (FGSC); however, the size of the generated PCR band is not uniform. Bands ranged from 350 to 500 bp based on the species. B, Amplification of the sequencecharacterized amplified region 85 region with the UBC85 primers, a 332-bp band obtained in the FGSC. C, PCR restriction fragment length polymorphism analysis of the translational elongation factor $1 \alpha$ gene that was amplified with the primer pair mh1/mh2; the generated 459-bp amplicons were subjected to Hpall-restriction digestion, and only F. graminearum sensu stricto (lanes 12 to 16) PCR amplicons produced a specific pattern consisting of two bands (199 and 260 bp). All other Fusarium spp., including the FGSC, did not produce this specific pattern and a single 450-bp band was observed. Using FG16F/R and UBC85 primers did not specifically differentiate $F$. graminearum sensu stricto from other species of the FGSC in addition to the presence of several false negatives within the FGSC group. Lane 1, F. oxysporum; lane 2, F. redolens; lane 3 , F. avenaceum; lane 4, F. acuminatum; lane 5, F. equiseti; lane 6, F. incarnatum; lane 7, F. poae; lane 8, F. culmorum; lanes 9 and 10 , F. cerealis; lane 11, F. mesoamericanum; lanes 12 to 16, F. graminearum sensu stricto; lane 17, F. boothii; lane 18, F. brasilicum; lane 19, F. meridionale; lane 20, F. nepalense; lane 21, F. vorosii; lane 22, F. gerlachii; lane 23, F. aethiopicum; and lane 24, F. acacia-mearnsii. 
produced a unique pattern to all $F$. graminearum sensu stricto isolates consisting of four fragments of $20+180+200+300 \mathrm{bp}$, while other Fusarium spp. produce a different restriction pattern consisting of three restriction fragments: $20+180+500 \mathrm{bp}$ (Supplementary Fig. $\mathrm{S} 1)$. For better visualization, a new primer pair, $\mathrm{mh} 1 / \mathrm{mh} 2$, that binds conserved sequences in all Fusarium spp. was designed to amplify a portion of the TEFl $\alpha$ gene with one HpaII restriction site in $F$. graminearum sensu stricto only. The $\mathrm{mh} 1$ and $\mathrm{mh} 2$ primers amplify a 459-bp PCR fragment from all Fusarium spp., and the restriction digestion of the $\mathrm{mh} 1 / \mathrm{mh} 2$ fragment produces a pattern unique to all $F$. graminearum sensu stricto isolates (two fragments: 199 bp + 260 bp), while any other Fusarium spp. were not affected by the HpaII enzyme and gave a 459-bp fragment (Fig. 2C). The newly designed PCR-RFLP marker accurately and selectively distinguishes $F$. graminearum sensu stricto from all other Fusarium spp., including members of FGSC. The capability of HpaII enzyme to digest its DNA target site in a short time has been investigated and results showed that $15 \mathrm{U}$ of $\mathrm{HpaII}$ can efficiently and rapidly digest approximately $400 \mathrm{ng}$ of $\mathrm{mh} 1 / \mathrm{mh} 2$ PCR amplicons in $10 \mathrm{~min}$ (Supplementary Fig. S2). The FastDigest HpaII enzyme (15 U) can also completely digest approximately $400 \mathrm{ng}$ of $\mathrm{hm} 1 / \mathrm{mh} 2$ PCR amplicons in 5 min (Fig. 2C).

Specificity and sensitivity of the $\mathrm{mh} 1 / \mathrm{mh} 2 \mathrm{PCR}$ primer pair. Specificity of the $\mathrm{mh} 1 / \mathrm{mh} 2$ primer pair was tested with several $F$ usarium spp. and all isolates showed strong PCR amplification and yielded a single band, with a 459-bp fragment from all tested Fusarium spp. No amplification occurred when the $\mathrm{mh} 1 / \mathrm{mh} 2$ primer pair was tested with gDNA from other fungal pathogens involved in FHB and FRR diseases in cereals and soybean, respectively, such as $C$. spaethianum and $R$. solani. Other fungal pathogens such as $S$. sclerotiorum and D. caulivora produced 500- and 550-bp bands, respectively, but HpaII did not cleave these bands. Sensitivity of the $\mathrm{mh} 1 / \mathrm{mh} 2$ primer pair was tested with different concentrations of pure $F$. graminearum sensu stricto gDNA templates (from $100 \mathrm{ng}$ down to $1 \mathrm{fg}$ ). The $\mathrm{mh} 1 / \mathrm{mh} 2$ primer pair, when used at $200 \mu \mathrm{M}$, can detect $F$. graminearum sensu stricto gDNA templates down to $10 \mathrm{pg}$.

In silico restriction digestion of the TEF1 $\alpha$ gene. During the current study, the newly designed PCR-RFLP marker was tested in vitro with 19 Fusarium spp. (Fig. 2). The in vitro results showed that the HpaII PCR-RFLP marker can efficiently detect $F$. graminearum sensu stricto species and differentiate this pathogen among closely related Fusarium spp. within the FGSC and other related Fusarium pathogens involved in soybean root rot and FHB (Table 1). To confirm the accuracy of the HpaII PCR-RFLP marker and its unique specificity to $F$. graminearum sensu stricto, more $T E F 1 \alpha$ sequences have been retrieved from GenBank to be analyzed for the presence or absence of the HpaII restriction site. TEF1 $\alpha$ sequences of species in Table 1 have been used as query to retrieve similar sequences to each species from different plant hosts in different geographic regions (Supplementary Table S1). In total, 85 Fusarium TEF1 $\alpha$ sequences were analyzed in silico with the RestrictionMapper program (version 3 ), and results confirmed that only mh1/mh2 PCR fragments from $F$. graminearum sensu stricto isolates (isolated from different hosts in different geographic regions) were digested with HpaII enzyme and produced the characteristic restriction pattern as obtained during the in vitro experiments $(199+260 \mathrm{bp})$. On the other hand, all other Fusarium spp. were not digested and a 450-bp PCR band was obtained. A phylogenetic tree was generated based on the TEF1 $\alpha$ sequences of different Fusarium spp. involved in FHB and FRR (Fig. $3)$. DNA sequence logos for the HpaII restriction site and flanking sequences (10 upstream and 10 downstream nucleotides) clearly showed that the HpaII restriction site is unique to all $F$. graminearum sensu stricto isolates and absent in all other Fusarium spp.

Detection of $F$. graminearum sensu stricto in infected plant tissues using the HpaII PCR-RFLP tool. Both F. cerealis (Carm1) and $F$. graminearum sensu stricto (Carm 3 ) were able to infect wheat and soybean and produce FHB and FRR symptoms in wheat and soybean, respectively. The presence of both $F$. graminearum sensu stricto and $F$. cerealis in infected plant tissues was confirmed by reisolation of the pathogen on PDA medium. The pathogens were reisolated from a subset of infected plants and identified morphologically as $F$. graminearum sensu stricto and $F$. cerealis. DNA was extracted from the reisolated Fusarium pathogens and TEF1 $\alpha$ sequences were found to be $100 \%$ identical to $F$. graminearum sensu stricto and $F$. cerealis. FRR symptoms in soybean root at $\mathrm{V} 1$ and $\mathrm{V} 3$ growth stages are shown in Figure 4A, and FHB symptoms at wheat at full maturation stage are shown in Figure 4B (see Supplementary Table S2 for FRR and FHB scores). The $\mathrm{mh} 1 / \mathrm{mh} 2$ primer pair was used to detect $F$. graminearum sensu stricto and $F$. cerealis in infected soybean and wheat plants. A 459-bp PCR amplicon was obtained using the $\mathrm{mh} 1 / \mathrm{mh} 2$ primer pair from soybean roots infected with $F$. graminearum sensu stricto at V1 and V3 growth stages, as

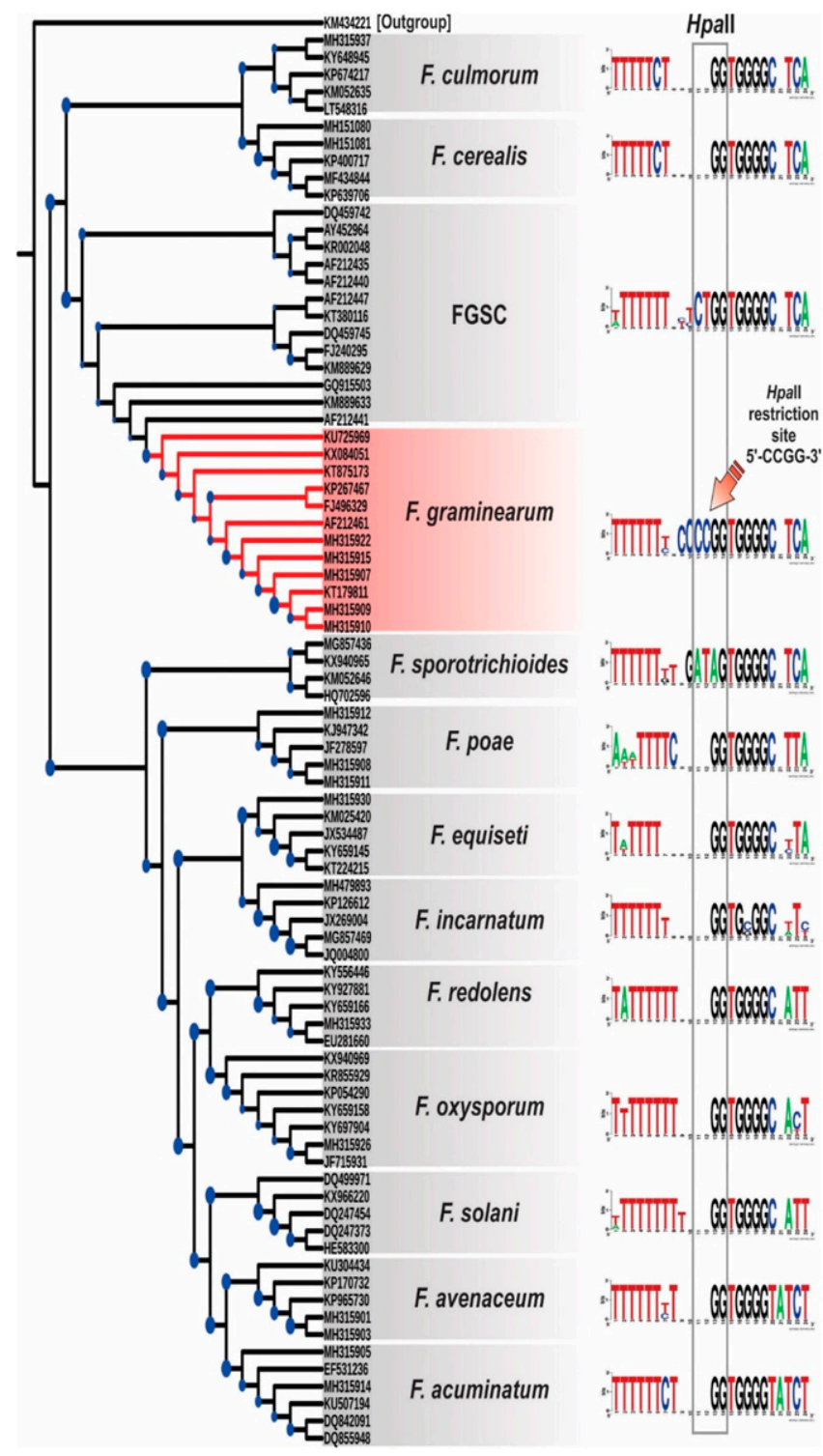

Fig. 3. Phylogenetic tree based on the translational elongation factor $1 \alpha(T E F 1 \alpha)$ sequences of different Fusarium spp. involved in Fusarium head blight and Fusarium root rot diseases in wheat and soybean, respectively. The tree topology based on neighbor-joining analysis and the largest and smallest circles on nodes represent bootstrap support values (100 to 1,000 respectively). Numbers in the tree represent the GenBank accession number of the TEF1 $\alpha$ nucleotide sequences used in the phylogenetic analysis. The tree is rooted with Fusarium pseudograminearum (KM434221). FGSC = F. graminearum species complex. Nucleotide sequence logos are shown for the Hpall restriction site and flanking sequences (10 upstream and 10 downstream nucleotides). The Hpall restriction site $\left(5^{\prime}-\mathrm{CCGG}-3^{\prime}\right)$ is boxed in gray-line box. Logos were generated by the online program WebLogo, version 2.8.2 (Crooks et al. 2004) (http://weblogo.berkeley. edu/logo.cgi). See Supplementary Table $\mathrm{S} 1$ for detailed information about the host plant and geographic region. 
well as from wheat spikes infected with the same pathogen. The same band was also obtained from soybean and wheat spikes infected with F. cerealis (Fig. 4C). The 459-bp bands from infected soybean and wheat tissues, when digested with HpaII, produced a single 459-bp band for $F$. cerealis. HpaII digested the $F$. graminearum sensu stricto band into two bands of 199 and 260 bp. The HpaII PCR-RFLP marker showed highly accurate detection of $F$. graminearum sensu stricto and was able to differentiate this pathogen from other closely related species.

$F$. graminearum sensu stricto specific primers. As mentioned above, the $\mathrm{Fg} 16 \mathrm{~F} / \mathrm{R}$ and the UBC58F/R primer pairs have been used to specifically detect members within the FGSC. During the current study, we attempted to validate these two commonly used primer pairs on a subset of Fusarium spp. but the results showed that neither primer pair could distinguish $F$. graminearum sensu stricto from other species in the FGSC (Fig. 2A and B). In addition, the two primer pairs can generate false negatives with several species and isolates within the FGSC, giving the possibility for misdetection of any of these Fusarium pathogens. During this study, sequence analysis of the TEF $1 \alpha$ gene in FGSC and other Fusarium spp. involved in FHB and FRR diseases to cereal and soybean crops, respectively, allowed for the design of two primers specific to $F$. graminearum sensu stricto (FgssF and FgssR). The unique HpaII restriction site was used to design these two primers, in which the $3^{\prime}$ end of each primer was designed to pair with the $5^{\prime}-\mathrm{CC}-3^{\prime}$ nucleotides in the enzyme's
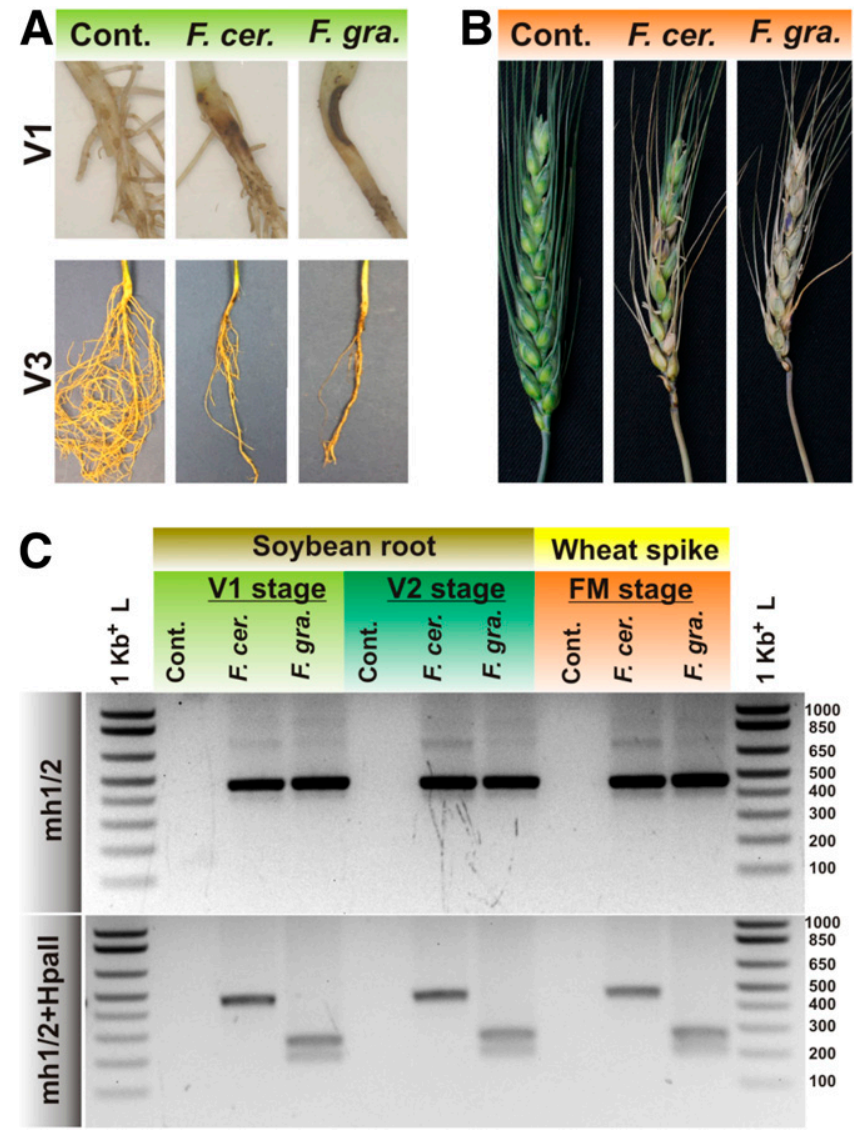

Fig. 4. Detection of Fusarium graminearum sensu stricto from infected soybean plants (with root rot symptoms) and infected wheat plants (with Fusarium head blight symptoms). A, Soybean plants infected with $F$ cerealis ( $F$. cer.) and $F$. graminearum sensu stricto in ( $F$. gar.) in V1 and V3 growth stages. Noninfected soybean plants were used as control (Cont.). B, Wheat spikes infected with $F$. cerealis and $F$. graminearum sensu stricto; noninfected wheat spikes were used as control. C, Detection of $F$. cerealis and F. graminearum sensu stricto in both soybean roots and wheat spikes using the $\mathrm{mh} 1 / \mathrm{mh} 2$ primer pair that produced 459 bp bands in both pathogens and no bands in the control. Only F. graminearum sensu stricto bands were recognized by the Hpall enzyme and produced two bands (199 and $260 \mathrm{bp}$ ). restriction site (5'-CCGG-3'). Sequence similarities within the TEF1 $\alpha$ gene within the genus Fusarium are extremely high, and the most effective way to design species-specific primers is to make the few nucleotides in the $3^{\prime}$ end of the primer specific to the species of interest. For successful DNA polymerase extension, the primer's $3^{\prime}$ end should be an exact match to the DNA template. Another important factor for DNA polymerase extension is to design the primer with $\mathrm{G}$ or $\mathrm{C}$ at the $3^{\prime}$ end for stronger binding. These two factors were considered when designing the FgssF and FgssR primers with the $5^{\prime}-\mathrm{CC}-3^{\prime} 3^{\prime}$ end that is unique and specific to $F$. graminearum sensu stricto. The FgssF primer was paired with ef 2 while the FgssR primer was paired with FusF to amplify 354- and 368-bp fragments, respectively, with all isolates of the $F$. graminearum sensu stricto, whereas no bands were generated with all other Fusarium spp. belonging to the FGSC or any other Fusarium sp.

Both primer pairs (FgssF/ef2 and FusF/FgssR) were found to have strong single bands and have high sensitivity and specificity to $F$. graminearum sensu stricto, and no bands were generated with any other Fusarium spp. belonging to the FGSC or other Fusarium spp. involved in FHB and FRR diseases in cereal and soybean crops, respectively (Fig. 5). Sensitivity of both FusF/FgssR and FgssF/ef2 primer pairs was tested with different concentrations of pure $F$. graminearum sensu stricto gDNA templates (from $10 \mathrm{ng}$ down to $100 \mathrm{fg}$ ). The FusF/FgssR primer pair, when used at $200 \mu \mathrm{M}$, can detect $F$. graminearum sensu stricto gDNA templates down to $100 \mathrm{fg}$, while sensitivity of FgssF/ef 2 primer pair was 1 pg (Fig. 5). We recommend using FusF/FgssR for specific detection of $F$. graminearum sensu stricto due to the higher sensitivity of this primer pair.

\section{Discussion}

Several approaches have been used for fungal classification and to define species limits of the genus Fusarium, in which classification based on morphology (i.e., known as "morphological species complex") was considered the main approach used to classify Fusarium spp. and was used by fungal taxonomists for over 200 years (Aoki et al. 2014; Cai et al. 2011; Nelson 1991; Summerell et al. 2010). Another concept is the "biological species concept", which describes species as groups of populations that interbreed with each other (Mayr 1940, 1963). There are limitations for both morphological and biological species concepts; in the former, there are not enough unique morphological characters for all species needed to be discriminated whereas, in the later, there are many Fusarium isolates that reproduce asexually and rarely produce sexual stages (Nelson 1991; Summerell et al. 2010). Recently, the "phylogenetic species concept", which defined species as the small monophyletic clade of organisms that share a derived character set (Cracraft 1983; Taylor et al. 2000), was developed and used to define species limits. This concept separates species based on converting differenced in DNA sequence of selected genes into quantitative measurements of genetic relatedness (Summerell et al. 2010). Approximately 300 phylogenetic species were identified within the genus Fusarium based on molecular phylogenetics (i.e., phylogenetic species concept). Using the phylogenetic species recognition criterion, O'Donnell et al. (2000) divided $F$. graminearum into seven phylogenetically distinct species based on phylogenetic analysis of six single-copy genes. Other studies based on high-throughput multilocus genotyping with DNA sequences from 13 nuclear genes divide $F$. graminearum into 16 monophyletic species. These species are collectively referred to as the FGSC (Cai et al. 2011; O'Donnell et al. 2004; Sarver et al. 2011; Starkey et al. 2007). F. graminearum sensu stricto is considered the main FHB causative agent within the FGSC ( $F$. graminearum sensu lato), and recently was found to show cross-pathogenicity and cause root rot of soybean. For this reason, specific detection of $F$. graminearum sensu stricto and differentiating this important toxigenic pathogen from other species in the FGSC and other closely related Fusarium spp. is considered an important issue for accurate disease diagnosis, better control, and minimizing the negative effects of mycotoxins. In the present study, we have developed two-step and one-step molecular tools to detect $F$. graminearum sensu stricto in vitro and in planta (i.e., infected wheat and soybean tissues) and 
differentiate this pathogen from other Fusarium pathogens involved in cereals and soybean diseases.

PCR-RFLP markers for Fusarium spp. PCR-RFLP markers based on ITS sequence variations have been developed to identify and study the genetic relationships between different Fusarium spp. (Cai et al. 2011; O'Donnell et al. 2004; Sarver et al. 2011; Starkey et al. 2007) The nuclear ribosomal ITS region has been used intensively as a marker for species identification since the development of the first universal primers to amplify this DNA region in fungi. Schoch et al. (2012) proposed using ITS as the sole universal barcode for fungi; however, the authors stated that this proposal will satisfy most but not all mycologists. Phylogenetically, closely related species (i.e., cryptic species such as the FGSC) differ by as little as as one to only a few nucleotide positions at the ITS level (Cai et al. 2011; O'Donnell et al. 2004; Sarver et al. 2011; Starkey et al. 2007). For this reason, Kiss (2012) did not consider ITS to be a sufficiently powerful tool to discriminate between closely related species.

The $\beta$-TUB which encodes for the structural proteins of microtubules and other structural components in eukaryotes was also used to differentiate between different plant and human pathogenic Fusarium spp. (O'Donnell et al. 2009; Yli-Mattila et al. 2004). Nosratabadi et al. (2018) evaluated the efficiency of PCR-RFLP based on the $\beta$-TUB region sequence to study genetic relationships in a total of 107 Fusarium isolates. The proposed PCR-RFLP protocol is based on amplifying the $\beta-T U B$ gene and digesting the amplified fragments with three restriction enzymes, which allowed differentiation between $F$. graminearum, $F$. culmorum, and $F$. cerealis but not between other investigated Fusarium spp. For this reason, Nosratabadi et al. (2018) recommend that other genes must be evaluated to overcome the limitations of the $\beta-T U B$ gene in differentiating these species.
The TEF $1 \alpha$ gene is considered the most frequently used sequence to define species boundaries within the genus Fusarium and has high phylogenetic utility because it is highly conserved and highly informative at the species level in Fusarium. In addition, no nonorthologous copies of this gene have been detected in the genus Fusarium. The Fusarium DNA sequence database (FUSARIUM-ID, v. 1.0), which is currently considered to be the most important tool for Fusarium sp. identification, is also based on TEFl $\alpha$ sequences (Geiser et al. 2004). The TEF $1 \alpha$ gene was first used as a phylogenetic marker in fungi by O'Donnell et al. (1998) to study the evolutionary origins of $F$. oxysporum f. sp. cubense, the causal pathogen of Panama disease of banana. They also developed degenerative primers (efl and ef2) based on conserved nucleotide sequences within the $T E F 1 \alpha$ gene shared by two ascomycetous fungi in the orders Hypocreales and Eurotiales, which can be used to partially amplify an approximately 700-bp fragment from the TEF $1 \alpha$ gene in a wide variety of ascomycetous fungi. The TEF $1 \alpha$ gene was used as a marker gene to study phylogenetic relationships within the genus Fusarium (Knutsen et al. 2004; Mbofung et al. 2007; O'Donnell et al. 2008) and also to detect and differentiate between different Fusarium spp. (Carbone and Kohn 1999; Garmendia et al. 2018; ZitnickAnderson et al. 2018). More interestingly, Kristensen et al. (2005), studied the relationship between Fusarium phylogeny and toxin production potential and found a correlation between major phylogenetic lineages and the ability to synthesize particular classes of toxins. Specific detection of $F$. graminearum sensu stricto is an extremely important issue due to the wide range of mycotoxins produced by isolates of this pathogen. Type-B trichothecenes are considered to be the most common mycotoxins produced by $F$. graminearum sensu stricto (DON, 15ADON, and 3ADON). The trichothecene chemotype produced by different species within the genus Fusarium seems to be dynamic and the pathogen can change this

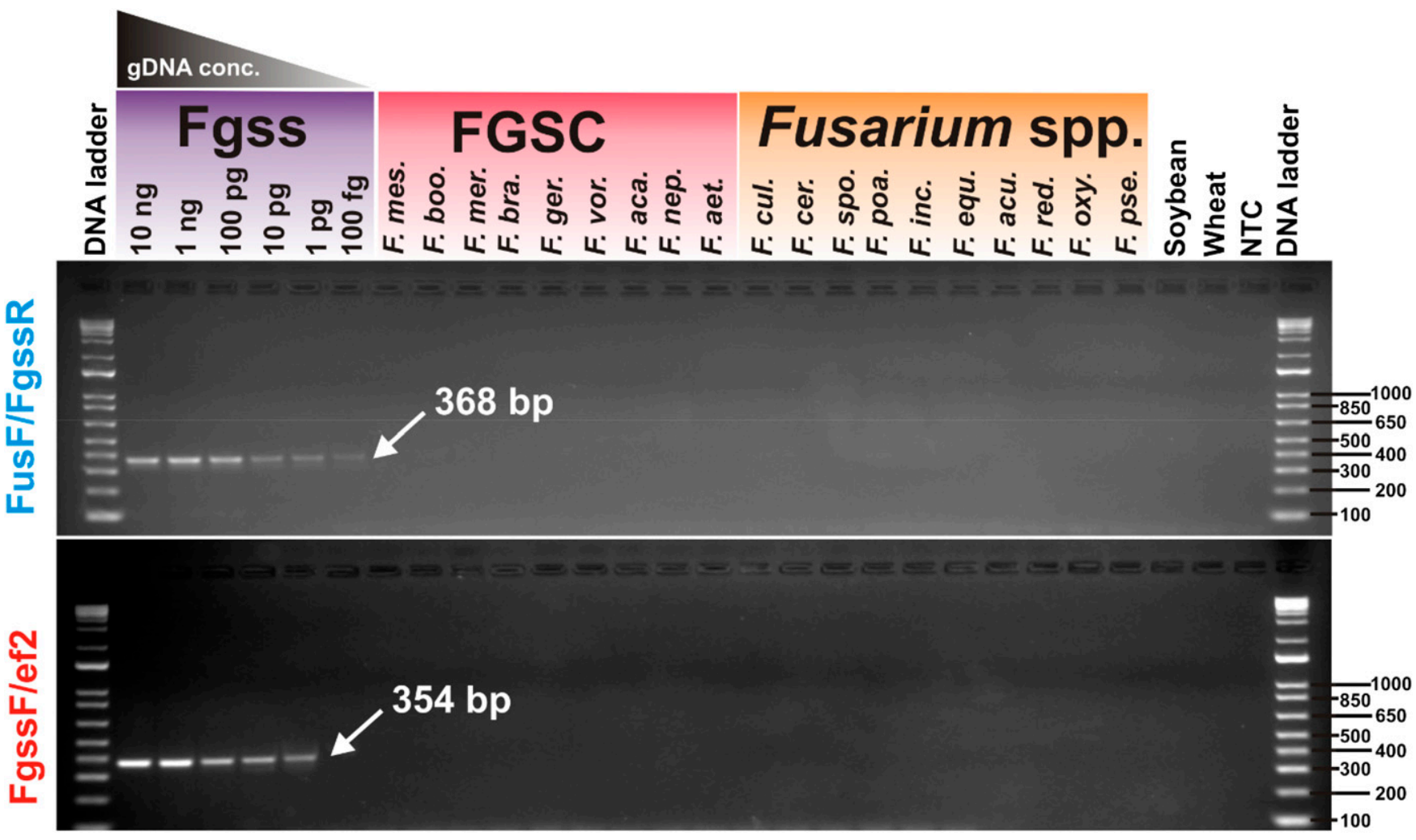

Fig. 5. Specific detection of Fusarium graminearum sensu stricto with FusF/FgssR and FgssF/ef2 primer pairs. The newly designed FgssR and FgssF primers were combined with FusF and ef2 primers to produce 368- or 354-bp fragments, respectively. Only F. graminearum sensu stricto (Fgss) isolates gave positive amplification whereas other species within the $F$. graminearum species complex (FGSC) or other closely related Fusarium spp. yielded no bands. Sensitivity of both primer pairs to different concentrations of pure $F$. graminearum sensu stricto (Fgss) genomic DNA (gDNA) templates (from $10 \mathrm{ng}$ down to $100 \mathrm{fg}$ ) is indicated. Both FusF/FgssR and FgssF/ef2 primer pairs also gave negative results with other fungal pathogens such as Rhizoctonia solani, Phytophthora infestans, Sclerotinia sclerotiorum, and Diaporthe caulivora (data not shown). No bands were obtained when gDNA from soybean and wheat was used as template. 
chemotype in response to surrounding biotic and abiotic factors. Recently, an $F$. graminearum population with $3 \mathrm{ADON}$ was found to replace the predominant 15DON population in Canada (Ward et al. 2008). More interestingly, a population of $F$. graminearum isolated from wheat in North America, which produced none of the known trichothecene mycotoxins despite causing normal FHB disease symptoms, has been identified. A new trichothecene chemotype (named NX-2) was characterized by liquid-chromatography tandem mass spectrometry. This new trichothecene mycotoxin is of type A due to the absence of the keto group at C-8 (Varga et al. 2015). This capability of $F$. graminearum sensu stricto to produce several toxins and even to switch from one chemotype to another obviously calls for an accurate specific detection. Species of the FGSC are undistinguishable morphologically and, due to their high similarity at the molecular level, there has been no specific molecular marker for $F$. graminearum sensu stricto Therefore, the PCR-RFLP tool and the species-specific PCR primers developed in the current study will be highly valuable in this regard.

Several TEFl $\alpha$-based PCR-RFLP tools have been developed to differentiate between different Fusarium spp. Nitschke et al. (2009) have conducted in silico analysis of TEF1 $\alpha$ sequences from 65 Fusarium isolates belonging to 18 species isolated from sugar beet. They developed a reliable PCR-RFLP tool to differentiate 17 of 18 species using two subsequent restriction digests of TEFl $\alpha$ PCR-amplified fragments. The study included $F$. graminearum and other Fusarium spp. involved in sugar beet diseases as well as other Fusarium spp. involved in other crops commonly grown with sugar beet in rotations such as wheat and corn; however, this study did not include any other FGSC representatives. Recently, a PCR-RFLP tool based on the TEF1 $\alpha$ sequence was developed to detect and differentiate $F$. graminearum sensu stricto from the remaining species within the FGSC and from other Fusarium spp. causing FHB in cereals (Garmendia et al. 2018). This PCR-RFLP tool was designed to amplify the $T E F 1 \alpha$ gene with the commonly used ef $1 / 2$ primer pair and digest the amplified PCR fragments with $B s a H I$ enzyme. The enzyme's restriction site (5'-GRCGYC-3', where, R could be A or G and $\mathrm{Y}$ could be $\mathrm{C}$ or $\mathrm{T}$ ) is only found in $F$. graminearum sensu stricto and is absent in other FGSC species and other closely related Fusarium spp. A unique pattern was only obtained with $F$. graminearum sensu stricto (two bands: 367 and 291 bp), whereas other Fusarium spp. were not cleaved. The multiple recognition sites for $B s a \mathrm{HI}\left(5^{\prime}\right.$ GRCGYC-3'; GACGCC, GACGTC, GGCGCC, and GGCGTC) affect its specificity to $F$. graminearum sensu stricto, and other Fusarium spp. could be recognized and cleaved by the $B s a \mathrm{HI}$ enzyme. We have analyzed the TEF1 $\alpha$ sequences from the FGSC and other closely related Fusarium spp. in silico, and results showed that several Fusarium spp. other than $F$. graminearum sensu stricto contain the restriction site of the BsaHI enzyme (5'-GRCGYC-3'). F. asiaticum (KY466738), F. culmorum (KY659120), and F. pseudograminearum (EF428635 and EF428644) have the exact restriction site of $B s a \mathrm{HI}\left(5^{\prime}\right.$-GGCGTC-3') at the same position and will give the same pattern as $F$. graminearum sensu stricto if tested with the BsaHI PCR-RFLP protocol. F. chlamydosporum (KY211036 and KF918546) also has a $B s a \mathrm{HI}$ restriction site (5'-GGCGCC-3') and will also give the same pattern as $F$. graminearum sensu stricto if tested with the $B s a \mathrm{HI}$ PCR-RFLP marker.

In the current study, the TEF $\alpha$ gene was amplified with the newly designed primers mh1 and mh2 to produce a 459-bp PCR amplicon. Subsequent RFLP to the PCR amplicons with HpaII restriction enzyme showed that only $F$. graminearum sensu stricto isolates were cleaved by HpaII to give a unique pattern specific for this important pathogen, consisting of two fragments (199 and $460 \mathrm{bp}$ ), whereas all other Fusarium spp. in the FGSC and other Fusarium spp. involved in FHB and FRR diseases in cereals and soybean, respectively, were not cleaved by the enzyme and a single band ( $459 \mathrm{bp}$ ) was obtained by these species. Additional in silico analysis for $84 T E F 1 \alpha$ gene sequences of $F$. graminearum sensu stricto and other species of the FGSC as well as other closely related Fusarium spp. isolated from different hosts in different places all over the world was conducted. The aim of this sequence analysis was to confirm that the HpaII restriction site is only found in $F$. graminearum sensu stricto and is absent in any other Fusarium sp. regardless of the geographic region and host. The results confirmed that the HpaII restriction site is unique to $F$. graminearum sensu stricto and can be considered a species-specific signature sequence that makes HpaII PCR-RFLP to TEF1 $\alpha$ PCR amplicons a suitable fast and accurate molecular tool to detect and differentiate $F$. graminearum sensu stricto from any other Fusarium spp.

F. graminearum sensu stricto-specific primers. Several molecular tools have been developed to detect and differentiate between species within the FGSC. Among these molecular tools, the Fg16F/R primer pair has been used extensively to detect FGSC members and differentiate between these closely related species by producing polymorphic PCR products (400 to $580 \mathrm{bp}$ ). The Fg16F and Fg16R primers have been used to study genetic diversity among different FGSC isolates, to differentiate between isolates within this species complex, and also to discriminate between the FGSC and other closely related Fusarium spp. Nicholson and coworkers (1998) initially used $\mathrm{Fg} 16 \mathrm{~F}$ and $\mathrm{Fg} 16 \mathrm{R}$ primers to differentiate between FGSC members and other closely related Fusarium spp. such as F. culmorum and $F$. crookwellense (syn. $F$. cerealis). PCR amplification using these primers produced polymorphic products (400 to $500 \mathrm{bp}$ ) with the FGSC but no products with DNA from other Fusarium spp. Carter et al. (2002) used the $\mathrm{Fg} 16 \mathrm{~F} / \mathrm{R}$ primer pair to study the genetic diversity among FGSC isolates and six SCAR groups have been obtained, each with a different PCR product size $(420,510,540$, 580, 520, and 400 bp for SCAR types 1 to 6 , respectively). Qu et al. (2008) have also used Fg16 F/R-based SCAR analysis to detect and differentiate between $F$. graminearum sensu stricto and $F$. asiaticum isolates. Two PCR product sizes were observed, corresponding to SCAR type 1 (420 bp: F. graminearum) and SCAR type 5 (520 bp: F. asiaticum) reported by Carter et al. (2002). The Fg16F and Fg16R primers are considered an important tool to detect FGSC isolates and differentiate between these important pathogens and other closely related Fusarium spp.; however, the Fg16F/R primer pair cannot specifically detect and differentiate between $F$. graminearum sensu stricto (the major causal agent of FHB of wheat worldwide) and other Fusarium spp. in the FGSC such as F. nepalense, F. gerlachii, and F. aethiopicum, because these species produce amplicons (420 bp) identical to those of $F$. graminearum sensu stricto when amplified with Fg16F/R. Other Fusarium spp. within the FGSC produce amplicons with $\mathrm{Fg} 16 \mathrm{~F} / \mathrm{R}$ that are very close in size (400 to $500 \mathrm{bp}$ ), which makes differentiation between species in this complex difficult. A recent study using representatives of all described FGSC species showed that, using the $\mathrm{Fg} 16 \mathrm{~F} / \mathrm{R}$ primer set to distinguish between some species in the FGSC, including $F$. graminearum sensu stricto, is difficult or impossible due to the narrow range of amplicon sizes obtained with this primer pair (Garmendia et al. 2018). The $\mathrm{UBC} 85 \mathrm{~F} / \mathrm{R}$ primer set is specific for FGSC and produces monomorphic amplicons (332 bp) (Schilling et al. 1996) with all $F$. graminearum sensu lato species. For this reason, this primer pair is not suitable to differentiate between species in the FGSC and also for specific detection of $F$. graminearum sensu stricto.

In the present work, specificity of the newly designed primer FgssR has been tested and results showed that this primer, when combined with the FusF primer, can only detect isolates of the F. graminearum sensu stricto and produce 368-bp amplicons, while all other Fusarium spp. in the FGSC produced no amplicons. The specificity of the FusF/FgssR primer pair was also tested with other Fusarium spp. involved in FHB and FRR in cereals and soybean, respectively, and results confirms the specificity of FusF/FgssR to $F$. graminearum sensu stricto. The HpaII PCR-RFLP and the FgssR-specific primer presented during the current work would effectively and accurately detect and differentiate $F$. graminearum sensu stricto from all other Fusarium spp., and can reduce time, effort, and cost in detecting this pathogen.

\section{Acknowledgments}

We thank D. McLaren, X. Wang, and M.-A. Henriquez from AAFC for providing us with some Fusarium cultures; R. Aboukhaddour, Lethbridge Research and 
Development Centre, AAFC for the use of cereal pathology lab facilities and $F$. pseudograminearum cultures to reconfirm the primers' specificity; the culture collection of the USDA-ARS for providing us with FGSC cultures; and the anonymous reviewers who provided valuable input.

\section{Literature Cited}

Abdelmagid, A., Hafez, M., Lawley, Y., Adam, L. R., and Daayf, F. 2018. First report of Fusarium cerealis causing root rot on soybean. Plant Dis. 102:2638.

Altschul, S. F., Gish, W., Miller, W., Myers, E. W., and Lipman, D. J. 1990. Basic local alignment search tool. J. Mol. Biol. 215:403-410.

Aoki, T., O'Donnell, K., and Geiser, D. M. 2014. Systematics of key phytopathogenic Fusarium species: Current status and future challenges. J. Gen. Plant Pathol. 80:189-201.

Aoki, T., Ward, T. J., Kistler, H. C., and O'Donnell, K. 2012. Systematics, phylogeny and trichothecene mycotoxin potential of Fusarium head blight cereal pathogens. JSM Mycotoxins 62:91-102.

Baird, S. M., and Bernard, E. C. 1984. Nematode population and community dynamics in soybean-wheat cropping and tillage regimes. J. Nematol. 16: 379-386.

Bajaj, R., Hu, W., Huang, Y., Chen, S., Prasad, R., Varma, A., and Bushley, K. E. 2015. The beneficial root endophyte Piriformospora indica reduces egg density of the soybean cyst nematode. Biol. Control 90:193-199.

Biazio, G. R. D., Leite, G. G. S., Tessmann, D. J., and Barbosa-Tessmann, I. P. 2008. A new PCR approach for the identification of Fusarium graminearum. Braz. J. Microbiol. 39:554-560.

Bienapfl, J. C., Malvick, D. K., and Percich, J. A. 2010. First report of Fusarium redolens causing root rot of soybean in Minnesota. Plant Dis. 94:1069.

Broders, K. D., Lipps, P. E., Paul, P. A., and Dorrance, A. E. 2007. Evaluation of Fusarium graminearum associated with corn and soybean seed and seedling disease in Ohio. Plant Dis. 91:1155-1160.

Cai, L., Giraud, T., Zhang, N., Begerow, D., Cai, G., and Shivas, R. G. 2011. The evolution of species concepts and species recognition criteria in plant pathogenic fungi. Fungal Divers. 50:121-133.

Carbone, I. and Kohn, L. M. 1999. A method for designing primer sets for speciation studies in filamentous ascomycetes. Mycologia 91:553-556.

Carter, J. P., Rezanoor, H. N., Holden, D., Desjardins, A. E., Plattner, R. D., and Nicholson, P. 2002. Variation in pathogenicity associated with the genetic diversity of Fusarium graminearum. Eur. J. Plant Pathol. 108:573-583.

Chiotta, M. L., Alaniz Zanon, M. S., Palazzini, J. M., Scandiani, M. M., Formento, A. N., Barros, G. G., and Chulze, S. N. 2016. Pathogenicity of Fusarium graminearum and $F$. meridionale on soybean pod blight and trichothecene accumulation. Plant Pathol. 65:1492-1497.

Cracraft, J. 1983. Species concepts and speciation analysis. Pages 159-187 in: Current Ornithology. R. F. Johnston, ed. Springer, Boston, MA, U.S.A.

Crooks, G. E., Hon, G., Chandonis, J. M., and Brenner, E. E. 2004. WebLogo: A sequence logo generator. Genome Res. 14:1188-1190.

David, R. F., BozorgMagham, A. E., Schmale, D. G., III, Ross, S. D., and Marr, L. C. 2014. Correlation and causality analyses of meteorological variables to examine predictors of Fusarium graminearum ascospore release. Pages 11-12 in: Proc. Natl. Fusarium Head Blight Forum-Parshall. St. Louis, MO, U.S.A.

Dill-Macky, R., and Jones, R. K. 2000. The effect of previous crop residues and tillage on Fusarium head blight of wheat. Plant Dis. 84:71-76.

Edel, V., Steinberg, C., Gautheron, N., and Alabouvette, C. 1997. Evaluation of restriction analysis of polymerase chain reaction (PCR)-amplified ribosomal DNA for the identification of Fusarium species. Mycol. Res. 101:179-187.

Ellis, M. L., Broders, K. D., Paul, P. A., and Dorrance, A. E. 2011. Infection of soybean seed by Fusarium graminearum and effect of seed treatments on disease under controlled conditions. Plant Dis. 95:401-407.

Engle, J. S., Lipps, P. E., and Mills, D. 2003. Fusarium Head Blight Severity Scale for Winter Wheat. Ext. Fact Sheet Bull. AC-48-03. The Ohio State University, Columbus, OH, U.S.A

Esmaeili Taheri, A., Hamel, C., Gan, Y., and Vujanovic, V. 2011. First report of Fusarium redolens from Saskatchewan and its comparative pathogenicity. Can. J. Plant Pathol. 33:559-564.

Garmendia, G., Umpierrez-Failache, M., Ward, T. J., and Vero, S. 2018. Development of a PCR-RFLP method based on the transcription elongation factor 1- $\alpha$ gene to differentiate Fusarium graminearum from other species within the Fusarium graminearum species complex. Food Microbiol. 70:28-32.

Garvey, G. S., McCormick, S. P., and Rayment, I. 2008. Structural and functional characterization of the TRI101 trichothecene 3-O-acetyltransferase from Fusarium sporotrichioides and Fusarium graminearum. J. Biol. Chem. 283:1660-1669.

Geiser, D. M., del Mar Jiménez-Gasco, M., Kang, S., Makalowska, I., Veeraraghavan, N., Ward, T. J., and O'Donnell, K. 2004. FUSARIUM-ID v. 1.0: A DNA sequence database for identifying Fusarium. Eur. J. Plant Pathol. 110:473-479.

Goswami, R. S., and Kistler, H. C. 2004. Heading for disaster: Fusarium graminearum on cereal crops. Mol. Plant Pathol. 5:515-525.

Gray, L. E., and Achenbach, L. A. 1996. Severity of foliar symptoms and root and crown rot of soybean inoculated with various isolates and inoculum rates of Fusarium solani. Plant Dis. 80:1197-1199.

Guenther, J. C., Hallen-Adams, H. E., Bücking, H., Shachar-Hill, Y., and Trail, F. 2009. Triacylglyceride metabolism by Fusarium graminearum during colonization and sexual development on wheat. Mol. Plant-Microbe Interact. $22: 1492-1503$

Guenther, J. C., and Trail, F. 2005. The development and differentiation of Gibberella zeae (anamorph: Fusarium graminearum) during colonization of wheat. Mycologia 97:229-237.

Guo, X. W., Fernando, W. G. D., Bullock, P., and Sapirstein, H. 2010. Quantifying cropping practices in relation to inoculum levels of Fusarium graminearum on crop stubble. Plant Pathol. 59:1107-1113.

Jurado, M., Vázquez, C., Patiño, B., and González-Jaén, M. T. 2005. PCR detection assays for the trichothecene-producing species Fusarium graminearum, Fusarium culmorum, Fusarium poae, Fusarium equiseti and Fusarium sporotrichioides. Syst. Appl. Microbiol. 28:562-568.

Kashyap, P. L., Rai, S., Kumar, S., Srivastava, A. K., Anandaraj, M., and Sharma, A. K. 2015. Mating type genes and genetic markers to decipher intraspecific variability among Fusarium udum isolates from pigeonpea. J. Basic Microbiol. 55:846-856.

Kimura, M., Matsumoto, G., Shingu, Y., Yoneyama, K., and Yamaguchi, I. 1998. The mystery of the trichothecene 3-O-acetyltransferase gene. FEBS Lett. 435: 163-168.

Kiss, L. 2012. Limits of nuclear ribosomal DNA internal transcribed spacer (ITS) sequences as species barcodes for fungi. Proc. Natl. Acad. Sci. U.S.A. 109:E1811

Knutsen, A. K., Torp, M., and Holst-Jensen, A. 2004. Phylogenetic analyses of the Fusarium poae, Fusarium sporotrichioides and Fusarium langsethiae species complex based on partial sequences of the translation elongation factor-1 alpha gene. Int. J. Food Microbiol. 95:287-295.

Kristensen, R., Mona, T. O. R. P., Kosiak, B., and Holst-Jensen, A. 2005 Phylogeny and toxigenic potential is correlated in Fusarium species as revealed by partial translation elongation factor 1 alpha gene sequences. Mycol. Res. 109:173-186

Kulik, T., Fordoński, G., Pszczółkowska, A., Płodzień, K., and Łapiński, M. 2004 Development of PCR assay based on ITS2 rDNA polymorphism for the detection and differentiation of Fusarium sporotrichioides. FEMS Microbiol. Lett. 239:181-186.

Lecomte, C., Alabouvette, C., Edel-Hermann, V., Robert, F., and Steinberg, C. 2016. Biological control of ornamental plant diseases caused by Fusarium oxysporum: A review. Biol. Control 101:17-30.

Letunic, I., and Bork, P. 2016. Interactive tree of life (iTOL) v3: An online tool for the display and annotation of phylogenetic and other trees. Nucleic Acids Res. 44:W242-W245

Liebman, M., and Dyck, E. 1993. Crop rotation and intercropping strategies for weed management. Ecol. Appl. 3:92-122.

Lu, C., Zhang, H., Wang, Y., and Zheng, X. 2015. Rapid diagnosis of Fusarium root rot in soybean caused by Fusarium equiseti or Fusarium graminearum using loop-mediated isothermal amplification (LAMP) assays. Australas. Plant Pathol. 44:437-443.

Mach, R. L., Kullnig-Gradinger, C. M., Farnleitner, A. H., Reischer, G., Adler, A., and Kubicek, C. P. 2004. Specific detection of Fusarium langsethiae and related species by DGGE and ARMS-PCR of a $\beta$-tubulin (tub1) gene fragment. Int. J. Food Microbiol. 95:333-339.

Marburger, D. A., Venkateshwaran, M., Conley, S. P., Esker, P. D., Lauer, J. G. and Ané, J. M. 2015. Crop rotation and management effect on Fusarium spp. populations. Crop Sci. 55:365-376.

Martinelli, J. A., Bocchese, C. A., Xie, W., O’Donnell, K., and Kistler, H. C. 2004 Soybean pod blight and root rot caused by lineages of the Fusarium graminearum and the production of mycotoxins. Fitopatol. Bras. 29:492-498.

Mayr, E. 1940. Speciation phenomena in birds. Am. Nat. 74:249-278.

Mayr, E. 1963. Animal Species and Evolution. Harvard University Press, Cambridge, MA, U.S.A.

Mbofung, G. Y., Hong, S. G., and Pryor, B. M. 2007. Phylogeny of Fusarium oxysporum $\mathrm{f}$. sp. lactucae inferred from mitochondrial small subunit, elongation factor $1-\alpha$, and nuclear ribosomal intergenic spacer sequence data. Phytopathology 97:87-98.

McMullen, M., Jones, R., and Gallenberg, D. 1997. Scab of wheat and barley: A re-emerging disease of devastating impact. Plant Dis. 81:1340-1348.

Nelson, P. E. 1991. History of Fusarium systematics. Phytopathology 81:1045-1048.

Nicholas, K. B., Nicholas, H. B., and Deerfield, D. W. 1997. GeneDoc: Analysis and visualization of genetic variation. EMBnet News 4:14.

Nicholson, P., Simpson, D. R., Weston, G., Rezanoor, H. N., Lees, A. K., Parry, D. W., and Joyce, D. 1998. Detection and quantification of Fusarium culmorum and Fusarium graminearum in cereals using PCR assays. Physiol. Mol. Plant Pathol. 53:17-37.

Niessen, L. 2007. PCR-based diagnosis and quantification of mycotoxin producing fungi. Int. J. Food Microbiol. 119:38-46.

Niessen, M. L., and Vogel, R. F. 1997. Specific identification of Fusarium graminearum by PCR with gaoA targeted primers. Syst. Appl. Microbiol. 20: 111-123.

Nitschke, E., Nihlgard, M., and Varrelmann, M. 2009. Differentiation of eleven Fusarium spp. isolated from sugar beet, using restriction fragment analysis of a polymerase chain reaction-amplified translation elongation factor $1 \alpha$ gene fragment. Phytopathology 99:921-929.

Nosratabadi, M., Kachuei, R., Rezaie, S. and Harchegani, A. B. 2018. Beta-tubulin gene in the differentiation of Fusarium species by PCR-RFLP analysis. Infez Med. 26:52-60. 
O’Donnell, K., Kistler, H. C., Cigelnik, E., and Ploetz, R. C. 1998. Multiple evolutionary origins of the fungus causing Panama disease of banana: Concordant evidence from nuclear and mitochondrial gene genealogies. Proc. Natl. Acad. Sci. U.S.A. 95:2044-2049.

O'Donnell, K., Kistler, H. C., Tacke, B. K., and Casper, H. H. 2000. Gene genealogies reveal global phylogeographic structure and reproductive isolation among lineages of Fusarium graminearum, the fungus causing wheat scab. Proc. Natl. Acad. Sci. U.S.A. 97:7905-7910.

O'Donnell, K., Sutton, D. A., Rinaldi, M. G., Gueidan, C., Crous, P. W., and Geiser, D. M. 2009. Novel multilocus sequence typing scheme reveals high genetic diversity of human pathogenic members of the Fusarium incarnatum$F$. equiseti and $F$. chlamydosporum species complexes within the United States. J. Clin. Microbiol. 47:3851-3861.

O'Donnell, K., Ward, T. J., Aberra, D., Kistler, H. C., Aoki, T., Orwig, N., and Klemsdal, S. S. 2008. Multilocus genotyping and molecular phylogenetics resolve a novel head blight pathogen within the Fusarium graminearum species complex from Ethiopia. Fungal Genet. Biol. 45:1514-1522.

O’Donnell, K., Ward, T. J., Geiser, D. M., Kistler, H. C., and Aoki, T. 2004. Genealogical concordance between the mating type locus and seven other nuclear genes supports formal recognition of nine phylogenetically distinct species within the Fusarium graminearum clade. Fungal Genet. Biol. 41:600-623.

Ortiz, C. S., Bell, A. A., Magill, C. W., and Liu, J. 2017. Specific PCR detection of Fusarium oxysporum f. sp. vasinfectum California race 4 based on a unique Tfol insertion event in the PHO gene. Plant Dis. 101:34-44.

Pioli, R. N., Mozzoni, L., Morandi, E. N., and Menard, M. 2004. First report of pathogenic association between Fusarium graminearum and soybean. Plant Dis. 88:220.

Placinta, C. M., D’Mello, J. P. F., and Macdonald, A. M. C. 1999. A review of worldwide contamination of cereal grains and animal feed with Fusarium mycotoxins. Anim. Feed Sci. Technol. 78:21-37.

Qu, B., Li, H. P., Zhang, J. B., Xu, Y. B., Huang, T., Wu, A. B., Zhao, C. S., Carter, J., Nicholson, P., and Liao, Y. C. 2008. Geographic distribution and genetic diversity of Fusarium graminearum and $F$. asiaticum on wheat spikes throughout China. Plant Pathol. 57:15-24.

Roy, K. W. 1997. Fusarium solani on soybean roots: Nomenclature of the causal agent of sudden death syndrome and identity and relevance of $F$. solani form B. Plant Dis. 81:259-266.

Sarver, B. A. J., Ward, T. J., Gale, L. R., Broz, K., Kistler, H. C., Aoki, T., Nicholson, P., Carter, J., and O'Donnell, K. 2011. Novel Fusarium head blight pathogens from Nepal and Louisiana revealed by multilocus genealogical concordance. Fungal Genet. Biol. 48:1096-1107.

Schilling, A. G., Moller, E. M., and Geiger, H. H. 1996. Polymerase chain reaction-based assays for species-specific detection of Fusarium culmorum, F. graminearum, and F. avenaceum. Phytopathology 86:515-522.

Schmale, D. G., III, and Ross, S. D. 2015. Highways in the sky: Scales of atmospheric transport of plant pathogens. Annu. Rev. Phytopathol. 53:591-611.

Schoch, C. L., Seifert, K. A., Huhndorf, S., Robert, V., Spouge, J. L., Levesque, C. A., and Miller, A. N. 2012. Nuclear ribosomal internal transcribed spacer (ITS) region as a universal DNA barcode marker for Fungi. Proc. Natl. Acad. Sci. U.S.A. 109:6241-6246.

Schreiber, M. M. 1992. Influence of tillage, crop rotation, and weed management on giant foxtail (Setaria faberi) population dynamics and corn yield. Weed Sci. 40:645-653.

Sella, L., Gazzetti, K., Castiglioni, C., Schäfer, W., and Favaron, F. 2014. Fusarium graminearum possesses virulence factors common to Fusarium head blight of wheat and seedling rot of soybean but differing in their impact on disease severity. Phytopathology 104:1201-1207.

Sindelar, A. J., Schmer, M. R., Jin, V. L., Wienhold, B. J., and Varvel, G. E. 2016. Crop rotation affects corn, grain sorghum, and soybean yields and nitrogen recovery. Agron. J. 108:1592-1602.
Stakheev, A. A., Khairulina, D. R., and Zavriev, S. K. 2016. Four-locus phylogeny of Fusarium avenaceum and related species and their species-specific identification based on partial phosphate permease gene sequences. Int. J. Food Microbiol. 225:27-37.

Starkey, D. E., Ward, T. J., Aoki, T., Gale, L. R., Kistler, H. C., Geiser, D. M., and O'Donnell, K. 2007. Global molecular surveillance reveals novel Fusarium head blight species and trichothecene toxin diversity. Fungal Genet. Biol. 44: 1191-1204.

Sudakin, D. L. 2003. Trichothecenes in the environment: Relevance to human health. Toxicol. Lett. 143:97-107.

Summerell, B. A., Laurence, M. H., Liew, E. C. Y., and Leslie, J. F. 2010 Biogeography and phylogeography of Fusarium: A review. Fungal Divers. 44:3-13.

Tan, M.-K., and Niessen, L. M. 2003. Analysis of rDNA ITS sequences to determine genetic relationships among, and provide a basis for simplified diagnosis of, Fusarium species causing crown rot and head blight of cereals Mycol. Res. 107:811-821.

Taylor, J. W., Jacobson, D. J., Kroken, S., Kasuga, T., Geiser, D. M., Hibbett D. S., and Fisher, M. C. 2000. Phylogenetic species recognition and species concepts in fungi. Fungal Genet. Biol. 31:21-32.

Thompson, J. D., Gibson, T. J., Plewniak, F., Jeanmougin, F., and Higgins, D. G. 1997. The Clustal X windows interface: Flexible strategies for multiple sequence alignment aided by quality analysis tools. Nucleic Acids Res. 25:4876-4882.

Trail, F., Xu, H., Loranger, R., and Gadoury, D. 2002. Physiological and environmental aspects of ascospore discharge in Gibberella zeae (anamorph Fusarium graminearum). Mycologia 94:181-189.

van der Lee, T., Zhang, H., van Diepeningen, A., and Waalwijk, C. 2015. Biogeography of Fusarium graminearum species complex and chemotypes: A review. Food Addit. Contam. Part A 32:453-460.

Varga, E., Wiesenberger, G., Hametner, C., Ward, T. J., Dong, Y., Schöfbeck, D., McCormick, S., Broz, K., Stückler, R., Schuhmacher, R., and Krska, R. 2015. New tricks of an old enemy: Isolates of Fusarium graminearum produce a type A trichothecene mycotoxin. Environ. Microbiol. 17:2588-2600.

Wang, J.-H., Ndoye, M., Zhang, J.-B., Li, H.-P., and Liao, Y.-C. 2011. Population structure and genetic diversity of the Fusarium graminearum species complex. Toxins (Basel) 3:1020-1037.

Ward, T. J., Clear, R. M., Rooney, A. P., O’Donnell, K., Gaba, D., Patrick, S., Starkey, D. E., Gilbert, J., Geiser, D. M., and Nowicki, T. W. 2008. An adaptive evolutionary shift in Fusarium head blight pathogen populations is driving the rapid spread of more toxigenic Fusarium graminearum in North America. Fungal Genet. Biol. 45:473-484.

Xue, A. G., Cober, E., Voldeng, H. D., Voldeng, H. D., Babcock, C., and Clear R. M. 2007. Evaluation of the pathogenicity of Fusarium graminearum and Fusarium pseudograminearum on soybean seedlings under controlled conditions. Can. J. Plant Pathol. 29:35-40.

Yli-Mattila, T., Gagkaeva, T., Ward, T. J., Aoki, T., Kistler, H. C., and O'Donnell, K. 2009. A novel Asian clade within the Fusarium graminearum species complex includes a newly discovered cereal head blight pathogen from the Russian Far East. Mycologia 101:841-852.

Yli-Mattila, T., Mach, R. L., Alekhina, I. A., Bulat, S. A., Koskinen, S., Kullnig Gradinger, C. M., and Klemsdal, S. S. 2004. Phylogenetic relationship of Fusarium langsethiae to Fusarium poae and Fusarium sporotrichioides as inferred by IGS, ITS, $\beta$-tubulin sequences and UP-PCR hybridization analysis. Int. J. Food Microbiol. 95:267-285.

Zarrin, M., Ganj, F., and Faramarzi, S. 2016. Analysis of the rDNA interna transcribed spacer region of the Fusarium species by polymerase chain reaction-restriction fragment length polymorphism. Biomed. Rep. 4:471-474.

Zitnick-Anderson, K., Simons, K., and Pasche, J. S. 2018. Detection and qPCR quantification of seven Fusarium species associated with the root rot complex in field pea. Can. J. Plant Pathol. 40:261-271. 